\title{
DNS study of dependence of bulk consumption velocity in a constant-density reacting flow on turbulence and mixture characteristics
}

\author{
Rixin $\mathrm{Yu}^{1, \mathrm{a})}$ and Andrei N. Lipatnikov ${ }^{2, \mathrm{~b})}$ \\ ${ }^{1}$ Division of Fluid Mechanics, Department of Energy Sciences, Lund University, 22100 Lund, Sweden \\ ${ }^{2}$ Department of Applied Mechanics, Chalmers University of Technology, Göteborg 412 96, Sweden
}

(Received 10 April 2017; accepted 16 June 2017; published online 30 June 2017)

\begin{abstract}
3D Direct Numerical Simulation (DNS) study of propagation of a single-reaction wave in forced, statistically stationary, homogeneous, isotropic, and constant-density turbulence was performed in order to evaluate both developing $U_{T}^{t}$ and fully developed $U_{T}^{s}$ bulk turbulent consumption velocities by independently varying a ratio of $0.5 \leq u^{\prime} / S_{L} \leq 90$ of the r.m.s. turbulent velocity to the laminar wave speed and a ratio of $0.39 \leq L_{11} / \delta_{F} \leq 12.5$ of the longitudinal integral length scale of the turbulence to the laminar wave thickness. Accordingly, the Damköhler $D a=\left(L_{11} S_{L}\right) /\left(u^{\prime} \delta_{F}\right)$ and Karlovitz $K a=\delta_{F} /\left(S_{L} \tau_{\eta}\right)$ numbers were varied from 0.01 to 24.7 and from 0.36 to 587 , respectively. Here, $\tau_{\eta}$ is the Kolmogorov time scale. The obtained DNS data show that, at sufficiently low $D a$, the fully developed ratio of $U_{T}^{s} / u^{\prime}$ is mainly controlled by $D a$ and scales as $\sqrt{D a}$. However, such a scaling should not be extrapolated to high $D a$. The higher $D a$ (or the lower $K a$ ), the less pronounced dependence of $U_{T}^{s} / u^{\prime}$ on a ratio of $L_{11} / \delta_{F}$. Moreover, scaling laws $U_{T} \propto u^{\prime \alpha} S_{L}^{1-\alpha}\left(L_{11} / \delta_{F}\right)^{\beta}$ are substantially different for developing $U_{T}^{t}$ and fully developed $U_{T}^{s}$, i.e., the scaling exponents $\alpha$ and, especially, $\beta$ depend on the wave-development time. Furthermore, $\alpha$ and, especially, $\beta$ depend on a method used to evaluate the developing $U_{T}^{t}$. Such effects can contribute to significant scatter of expressions for $U_{T}$ or $S_{T}$ as a function of $\left\{u^{\prime}, S_{L}, L_{11}, \delta_{F}\right\}$, obtained by parameterizing various experimental databases. Published by AIP Publishing. [http://dx.doi.org/10.1063/1.4990836]
\end{abstract}

\section{INTRODUCTION}

As reviewed elsewhere, ${ }^{1-4}$ turbulent flame speed $S_{T}$ and consumption (or burning) velocity $U_{T}$ were in the focus of experimental and theoretical research into premixed turbulent combustion for decades. Moreover, several approaches to unsteady multi-dimensional numerical simulations of premixed flames ${ }^{4-10}$ straightforwardly invoke a model expression for $U_{T}$ or $S_{T}$. However, ranking such expressions using experimental databases is substantially impeded because the vast majority of models of $U_{T}$ or $S_{T}$ address the simplest case of single-step chemistry and a constant-density flow, whereas experiments deal with complex chemistry flames characterized by significant density variations. Therefore, in order to properly assess various models of turbulent flame speed and consumption velocity, there is a fundamental need for data on $U_{T}$ and $S_{T}$ that are obtained under conditions consistent with assumptions invoked by the models, i.e., single-step chemistry and constant density. Direct Numerical Simulation (DNS) offers a unique opportunity to encompass such a database, and the major goals of the present work consist in (i) evaluating turbulent consumption velocities in DNSs of propagation of a single-reaction wave in constant-density turbulence, performed for a wide range of basic mixture and turbulence characteristics, and (ii) analyzing this database.

\footnotetext{
a) Electronic mail: rixin.yu@energy.lth.se

b) Electronic mail: andrei.lipatnikov@chalmers.se
}

Another goal of the present study stems from the following reasoning. Experimental data on $U_{T}$ and $S_{T}$ reported by different research groups are well known to yield significantly different values of these quantities, e.g., see Fig. 4.11 in Ref. 10 . As shown in many studies ${ }^{11-17}$ and reviewed elsewhere, ${ }^{4,10,18}$ the scatter of available experimental data on $U_{T}$ and $S_{T}$ results, at least in part, from sensitivity of these quantities to methods used to process raw experimental data. Indeed, the speed $S_{T}$ of a statistically stationary turbulent flame is commonly determined as follows: ${ }^{1,4,10,18} \rho_{u} S_{T}=\overline{\rho u}$, where $\rho$ is the mixture density, subscript $u$ designates the unburned gas, $u$ is the flow velocity in the direction normal to the mean flame brush, and $\bar{q}$ is the Reynolds-averaged value of a quantity $q$. However, in a typical statistically stationary turbulent flame such as V-shaped, Bunsen, or stagnation-point flame, the incoming mean flow of unburned gas is spatially non-uniform and the magnitude of the mean normal flux $\overline{\rho u}$ depends substantially on the choice of an iso-scalar surface whose speed is associated with $S_{T}$. For instance, the flux $\overline{\rho u}$ decreases significantly (by a factor of three or even larger ${ }^{11,12}$ ) from the leading to the trailing edge of a mean flame brush stabilized in a spatially diverging mean flow of unburned gas.

Moreover, if a mean flame brush is curved, areas of various mean iso-scalar surfaces within the flame brush will be different. Accordingly, the measured value of turbulent burning velocity $U_{T}$ depends substantially on the choice of the iso-scalar surface whose area is used to normalize the total burning rate when evaluating $U_{T}$. For instance, in a cylindrical Bunsen flame, the mean iso-scalar surface area measured 
close to the leading edge of the mean flame brush may be significantly (by a factor of two or even larger ${ }^{16}$ ) smaller than the mean iso-scalar surface area measured close to the trailing edge of the flame brush. Consequently, $U_{T}$ evaluated at the leading edge may be significantly larger than $U_{T}$ evaluated at the trailing edge.

In typical experimental studies of premixed turbulent combustion, such flow-divergence and mean-surfacecurvature effects are well pronounced due to significant thickness of the turbulent flame brush when compared to the thin laminar flame. Recently, Verma and Lipatnikov ${ }^{17}$ numerically showed that these effects can also cause well documented scatter ${ }^{4}$ of scaling exponents for $U_{T}$ or $S_{T}$ as a function of the basic mixture and turbulence characteristics.

However, it may not be necessarily true that the scatter of available experimental data on $U_{T}$ and $S_{T}$ is solely controlled by the two effects of flow-divergence and mean-surfacecurvature. The present study aims at showing two more effects that can substantially contribute to the scatter of the scaling exponents for $U_{T}$ or $S_{T}$ as a function of the basic mixture and turbulence characteristics. This goal is feasible because the well-known flow-divergence and mean-surface-curvature effects vanish in the case simulated in this work, i.e., propagation of a statistically planar reaction wave in homogeneous, isotropic, constant-density turbulence.

While the problem addressed in the present work is straightforwardly relevant to premixed turbulent combustion, the problem studied in this work is substantially simplified, e.g., because thermal expansion phenomena can significantly affect turbulent flames. Such effects were hypothesized in the pioneering work by Karlovitz et al. ${ }^{19}$ and Scurlock and Grover $^{20}$ and were clearly shown in recent papers, ${ }^{21-25}$ as well as in earlier contributions reviewed elsewhere. ${ }^{26-29}$

One reason for invoking the aforementioned simplifications consists in obtaining DNS database for assessing models under conditions that are consistent with assumptions invoked by the models, as already explained.

Another reason consists of a fact that propagation of a passive reaction wave in constant-density turbulence is still the corner-stone paradigm in research into premixed turbulent combustion. A number of advanced models and concepts are based on this corner-stone paradigm, with the thermal expansion phenomena and complex combustion chemistry being considered to supplement and expand, but not to revoke it. For instance, widely used combustion regime diagrams $7,30-32$ were developed within the framework of this paradigm. Therefore, the problem addressed in the present work appears to be of a general interest for the theory of turbulent reacting flows and of a particular interest for gaining fundamental insight into the influence of turbulence on premixed combustion. Nevertheless, the fact that thermal expansion and complex chemistry phenomena are beyond the scope of the present study should be borne in mind when applying the obtained results to premixed turbulent flames.

The paper is organized as follows. In Secs. II and III, governing equations and numerical methods are briefly described, while the reader interested in a more detailed discussion is referred to our recent papers. ${ }^{33,34}$ Conditions of the performed simulations are discussed in detail in Sec. IV. Results obtained for fully developed and developing turbulent consumption velocities are reported in Secs. V A and V B, respectively, followed by conclusions.

\section{GOVERNING EQUATIONS}

Constant-density turbulent flow is described by the continuity and Navier-Stokes equations

$$
\begin{gathered}
\nabla \cdot \mathbf{u}=0, \\
\frac{\partial \mathbf{u}}{\partial t}+(\mathbf{u} \cdot \nabla) \mathbf{u}=-\rho^{-1} \nabla p+v \nabla^{2} \mathbf{u}+\mathbf{f},
\end{gathered}
$$

where $t$ is the time, $\mathbf{u}$ is the flow velocity vector, $v$ and $p$ are the kinematic viscosity and pressure, respectively, and a vector-function $\mathbf{f}$ is added in order to maintain constant turbulence intensity by using energy forcing at low wavenumbers, as discussed in Sec. III.

Propagation of a reaction wave of non-zero thickness is modeled by the following convection-diffusion-reaction equation:

$$
\frac{\partial c}{\partial t}+\mathbf{u} \cdot \nabla c=D \nabla^{2} c+W
$$

for a scalar field $c$, which is equal to zero and unity in fresh reactants and products, respectively. The molecular diffusivity $D$ is set to be constant and the reaction rate

$$
W=\frac{1}{1+\tau} \frac{1-c}{\tau_{R}} \exp \left[-\frac{Z e(1+\tau)^{2}}{\tau(1+\tau c)}\right]
$$

depends on $c$ in a highly non-linear manner, similarly to the temperature-dependence of heat-release rate $\omega$ in flames. Here, $\tau_{R}$ is a constant reaction time scale, $\tau=6$, and parameter $Z e$, called the Zeldovich number via an analogy with premixed combustion, is specified in Sec. IV, see the eighth column in Table I therein. To further draw the analogy, it is worth noting that $(1+\tau)$ is associated with the density ratio $\rho_{u} / \rho_{b}$. The scalar $c$ is associated with the combustion progress variable, which is equal to $1-Y / Y_{u}=\left(T-T_{u}\right) /\left(T_{b}-T_{u}\right)$ in a premixed flame if the Lewis number $L e=a / D=1$. Here, $Y$ is the mass fraction of the deficient reactant, $T$ is the temperature, $a$ is the molecular heat diffusivity of the mixture, and subscript $b$ designates the burned products in the flame.

Premixed turbulent flames are commonly characterized using (i) a ratio $u^{\prime} / S_{L}$ of the r.m.s. turbulent velocity $u^{\prime}$ to the laminar flame speed $S_{L}$ and (ii) a ratio of an integral length scale of turbulence to a laminar flame thickness $\delta_{F}=D / S_{L}$. Accordingly, DNS cases were set up (i) by specifying $Z e$, $S_{L}$, and $\delta_{F}$ and (ii) by finding $D$ and $\tau_{R}$ that are required to obtain the specified $S_{L}$ and $\delta_{F}$ in pre-simulations of a planar 1D laminar reaction wave modeled with Eqs. (3) and (4).

\section{NUMERICAL METHOD}

The major difference between the present work and our earlier DNS studies ${ }^{33,34}$ consists in substituting the level set $(G)$ equation ${ }^{7,31}$ with Eq. (3), whereas numerical methods were basically similar in both computations. Accordingly, we will restrict ourselves to a very brief summary of the numerical methods and refer the interested reader to recent papers ${ }^{33,34}$ for more details. 
TABLE I. Studied DNS cases.

\begin{tabular}{|c|c|c|c|c|c|c|c|c|c|c|c|c|c|c|c|c|}
\hline & $\frac{\eta}{\Delta x}$ & $\frac{\Lambda_{x}}{\Lambda}$ & $N_{x}$ & $\frac{\Lambda}{\Lambda_{256}}$ & $\frac{L_{11}}{\Lambda}$ & $\frac{\tau_{t}}{\tau_{\eta}}$ & $Z e$ & $S c$ & $D a$ & Ka & $R e_{t}$ & $P e$ & $\frac{u^{\prime}}{S_{L}}$ & $\frac{L_{11}}{\delta_{F}}$ & $\frac{U_{T}^{s}}{u^{\prime}}$ & $\frac{U_{T}^{s}}{S_{L}}$ \\
\hline B1 & 0.68 & 4 & 256 & 1 & 0.13 & 5.5 & 6.0 & 0.04 & 4.09 & 1.34 & 26 & 1.02 & 0.5 & 2.1 & 2.18 & 1.09 \\
\hline B2 & 0.68 & 4 & 256 & 1 & 0.13 & 5.5 & 6.0 & 0.08 & 2.04 & 2.69 & 26 & 2.04 & 1. & 2.1 & 1.31 & 1.31 \\
\hline B3 & 0.68 & 4 & 256 & 1 & 0.13 & 5.5 & 6.0 & 0.16 & 1.02 & 5.38 & 26 & 4.09 & 2. & 2.1 & 0.89 & 1.78 \\
\hline B4 & 0.68 & 4 & 256 & 1 & 0.13 & 5.5 & 6.0 & 0.39 & 0.41 & 13.4 & 26 & 10.2 & 5. & 2.1 & 0.57 & 2.85 \\
\hline B5 & 0.68 & 4 & 256 & 1 & 0.13 & 5.5 & 6.0 & 0.78 & 0.20 & 26.9 & 26 & 20.4 & 10 & 2.1 & 0.40 & 4.0 \\
\hline B6 & 0.87 & 4 & 512 & 2 & 0.12 & 6.3 & 6.0 & 0.04 & 7.50 & 0.84 & 48 & 1.87 & 0.5 & 3.7 & 2.45 & 1.23 \\
\hline B7 & 0.87 & 4 & 512 & 2 & 0.12 & 6.3 & 6.0 & 0.08 & 3.75 & 1.67 & 48 & 3.75 & 1. & 3.7 & 1.69 & 1.69 \\
\hline B8 & 0.87 & 4 & 512 & 2 & 0.12 & 6.3 & 6.0 & 0.16 & 1.87 & 3.34 & 48 & 7.50 & 2. & 3.7 & 1.27 & 2.54 \\
\hline B9 & 0.87 & 4 & 512 & 2 & 0.12 & 6.3 & 6.0 & 0.39 & 0.75 & 8.36 & 48 & 18.7 & 5. & 3.7 & 0.80 & 4.0 \\
\hline B10 & 0.87 & 4 & 512 & 2 & 0.12 & 6.3 & 6.0 & 0.78 & 0.38 & 16.7 & 48 & 37.5 & 10 & 3.7 & 0.55 & 5.5 \\
\hline B11 & 1.07 & 4 & 1024 & 4 & 0.11 & 7.4 & 6.0 & 0.04 & 13.5 & 0.55 & 86 & 3.37 & 0.5 & 6.7 & 2.77 & 1.39 \\
\hline B12 & 1.07 & 4 & 1024 & 4 & 0.11 & 7.4 & 6.0 & 0.08 & 6.74 & 1.10 & 86 & 6.74 & 1. & 6.7 & 2.06 & 2.06 \\
\hline B13 & 1.07 & 4 & 1024 & 4 & 0.11 & 7.4 & 6.0 & 0.16 & 3.37 & 2.21 & 86 & 13.5 & 2. & 6.7 & 1.61 & 3.22 \\
\hline B14 & 1.07 & 4 & 1024 & 4 & 0.11 & 7.4 & 6.0 & 0.39 & 1.35 & 5.52 & 86 & 33.7 & 5. & 6.7 & 1.11 & 5.55 \\
\hline B15 & 1.07 & 4 & 1024 & 4 & 0.11 & 7.4 & 6.0 & 0.78 & 0.67 & 11.0 & 86 & 67.4 & 10. & 6.7 & 0.76 & 7.61 \\
\hline L1 & 1.32 & 4 & 1024 & 4 & 0.19 & 8.9 & 6.0 & 0.04 & 24.7 & 0.36 & 158 & 6.18 & 0.5 & 12.4 & 2.99 & 1.5 \\
\hline L2 & 1.32 & 4 & 1024 & 4 & 0.19 & 8.9 & 6.0 & 0.08 & 12.4 & 0.72 & 158 & 12.4 & 1. & 12.4 & 2.23 & 2.23 \\
\hline L3 & 1.32 & 4 & 1024 & 4 & 0.19 & 8.9 & 6.0 & 0.16 & 6.18 & 1.44 & 158 & 24.7 & 2. & 12.4 & 1.79 & 3.57 \\
\hline L4 & 1.32 & 4 & 1024 & 4 & 0.19 & 8.9 & 6.0 & 0.39 & 2.47 & 3.60 & 158 & 61.8 & 5. & 12.4 & 1.40 & 7.02 \\
\hline L5 & 1.32 & 4 & 1024 & 4 & 0.19 & 8.9 & 6.0 & 0.78 & 1.24 & 7.21 & 158 & 124. & 10. & 12.4 & 1.02 & 10.2 \\
\hline D1 & 1.08 & 8 & 2048 & 4 & 0.11 & 7.5 & 6.0 & 0.78 & 0.17 & 43.3 & 89 & 69.3 & 20 & 3.46 & 0.38 & 7.69 \\
\hline D2 & 1.08 & 8 & 2048 & 4 & 0.11 & 7.5 & 6.0 & 0.78 & 0.08 & 97.4 & 90 & 70.6 & 30. & 2.35 & 0.25 & 7.58 \\
\hline D3 & 1.08 & 8 & 2048 & 4 & 0.11 & 7.5 & 6.0 & 0.78 & 0.04 & 173. & 90 & 70.4 & 40 & 1.76 & 0.19 & 7.48 \\
\hline D4 & 1.08 & 8 & 2048 & 4 & 0.11 & 7.5 & 6.0 & 0.78 & 0.02 & 390. & 89 & 69.5 & 60. & 1.16 & 0.13 & 7.59 \\
\hline D5 & 0.88 & 8 & 1024 & 2 & 0.12 & 6.4 & 6.0 & 0.08 & 0.97 & 6.53 & 50 & 3.90 & 2. & 1.95 & 0.88 & 1.76 \\
\hline D6 & 0.88 & 8 & 1024 & 2 & 0.12 & 6.4 & 6.0 & 0.08 & 0.16 & 40.8 & 50 & 3.90 & 5. & 0.78 & 0.35 & 1.73 \\
\hline D7 & 0.88 & 8 & 1024 & 2 & 0.12 & 6.4 & 6.0 & 0.08 & 0.04 & 163. & 50 & 3.90 & 10. & 0.39 & 0.17 & 1.73 \\
\hline D8 & 0.88 & 8 & 1024 & 2 & 0.12 & 6.4 & 6.0 & 0.78 & 0.04 & 147 & 50 & 38.8 & 30. & 1.29 & 0.19 & 5.69 \\
\hline D9 & 0.88 & 8 & 1024 & 2 & 0.12 & 6.4 & 6.0 & 0.78 & 0.01 & 587 & 50 & 39.1 & 60. & 0.65 & 0.09 & 5.69 \\
\hline D10 & 0.69 & 8 & 512 & 1 & 0.13 & 5.5 & 6.0 & 0.78 & 0.02 & 238 & 27 & 20.9 & 30. & 0.70 & 0.14 & 4.19 \\
\hline $\mathrm{T} 1$ & 1.07 & 4 & 1024 & 4 & 0.11 & 7.4 & 6.0 & 0.01 & 4.11 & 1.81 & 86 & 1.03 & 0.5 & 2.1 & 2.19 & 1.09 \\
\hline $\mathrm{T} 2$ & 1.07 & 4 & 1024 & 4 & 0.11 & 7.4 & 6.0 & 0.24 & 0.21 & 36.2 & 86 & 20.5 & 10. & 2.1 & 0.42 & 4.18 \\
\hline $\mathrm{T} 3$ & 1.07 & 8 & 2048 & 4 & 0.11 & 7.5 & 6.0 & 3.13 & 0.08 & 97.4 & 90 & 281. & 60. & 4.67 & 0.25 & 15.1 \\
\hline $\mathrm{T} 4$ & 1.07 & 8 & 2048 & 4 & 0.11 & 7.5 & 6.0 & 7.04 & 0.08 & 97.6 & 89 & 626. & 90. & 6.94 & 0.25 & 22.8 \\
\hline T5 & 1.07 & 4 & 1024 & 4 & 0.11 & 7.4 & 6.0 & 0.03 & 0.67 & 11.0 & 89 & 2.68 & 2. & 1.34 & 0.76 & 1.51 \\
\hline T6 & 1.07 & 4 & 1024 & 4 & 0.11 & 7.4 & 6.0 & 0.20 & 0.68 & 11.0 & 89 & 16.9 & 5. & 3.39 & 0.76 & 3.78 \\
\hline K1 & 0.87 & 4 & 512 & 2 & 0.12 & 6.3 & 6.0 & 0.04 & 1.89 & 3.33 & 48 & 1.89 & 1. & 1.89 & 1.29 & 1.29 \\
\hline $\mathrm{K} 2$ & 1.07 & 4 & 1024 & 4 & 0.11 & 7.4 & 6.0 & 0.02 & 1.70 & 4.41 & 87 & 1.70 & 1. & 1.69 & 1.25 & 1.25 \\
\hline K3 & 2.70 & 8 & 2048 & 4 & 0.13 & 5.7 & 6.0 & 28.2 & 0.09 & 62.1 & 26 & 744. & 90. & 8.27 & 0.27 & 24.3 \\
\hline K4 & 2.05 & 8 & 1024 & 2 & 0.14 & 5.3 & 6.0 & 3.13 & 0.01 & 431. & 14 & 44.0 & 60. & 0.73 & 0.10 & 6.06 \\
\hline $\mathrm{H} 1$ & 2.69 & 4 & 1024 & 1 & 0.13 & 5.7 & 6.0 & 0.78 & 0.21 & 27.7 & 26 & 20.7 & 10. & 2.1 & 0.41 & 4.09 \\
\hline $\mathrm{H} 2$ & 2.69 & 4 & 1024 & 1 & 0.13 & 5.7 & 17.1 & 0.78 & 0.21 & 27.7 & 26 & 20.7 & 10. & 2.1 & 0.41 & 4.07 \\
\hline $\mathrm{H} 3$ & 1.07 & 4 & 1024 & 4 & 0.11 & 7.4 & 17.1 & 0.24 & 0.21 & 36.2 & 86 & 20.5 & 10. & 2.1 & 0.41 & 4.11 \\
\hline $\mathrm{H} 4$ & 1.07 & 8 & 2048 & 4 & 0.11 & 7.5 & 17.1 & 0.78 & 0.02 & 389. & 90 & 70.1 & 60. & 1.16 & 0.12 & 7.37 \\
\hline H5 & 0.88 & 4 & 1024 & 4 & 0.06 & 6.2 & 6.0 & 0.78 & 0.39 & 16.1 & 49 & 38.7 & 10. & 3.88 & 0.57 & 5.70 \\
\hline
\end{tabular}

The computational domain is a fully periodic rectangular box of size of $\Lambda_{x} \times \Lambda \times \Lambda$. It is discretized using a uniform staggered Cartesian grid of $N_{x} \times N \times N$ cells with $N_{x}=N \Lambda_{x} / \Lambda$. Therefore, spatial resolution $\Delta x=\Lambda_{x} / N_{x}=\Lambda / N=\Delta y=\Delta z$ is the same in the axial ( $x$ ) and transverse ( $y$ and $z$ ) directions.

Boundary conditions are periodic in all three directions, thus, enabling a piece of a wave surface that comes to the left boundary $(x=0)$ at certain $t, y$, and $z$ to enter the computational domain through the right boundary $\left(x=\Lambda_{x}\right)$ at the same $t, y$, and $z$, respectively. Such a method allows us to strongly improve sampling statistics by simulating a number of cycles of wave propagation through the computational domain. This method is only justified if the reaction affects neither the density nor the viscosity. Otherwise, wave surfaces that come to $x=0$ and enter through $x=\Lambda_{x}$ will be affected by flow fields characterized by different $\rho$ and/or $v$.

Simulations are performed using a simplified in-house DNS solver ${ }^{35}$ developed for low Mach number reacting flows and equipped with a standalone stiff chemistry solver for a general kinetic mechanism. The solver was already applied to various reacting flow systems. ${ }^{36-39}$ In the original solver, the temporal integration of the governing equations is based on a second order symmetrical Strang splitting algorithm. In the present work, the solver is simplified because the source 
term $W(c)$ given by Eq. (4) is not stiff. In particular, when numerically integrating Eq. (3), temporal advancement is performed for a full time-step $\left(\Delta t=t^{n+1}-t^{n}=0.029 \Delta x / u^{\prime}\right)$ using the Adams-Bashforth method in multiple sub-time steps, i.e., $\Delta t_{*}=\Delta t / K=t_{*}^{k+1}-t_{*}^{k}, k=0, \ldots, K, t_{*}^{0}=t^{n}$, and $K=1+$ floor $\left[\Delta t \cdot \mathcal{D}(\Delta x)^{-2} / 0.075\right]$, where floor is the integer floor function. Accordingly,

$$
\begin{aligned}
\frac{c\left(t_{*}^{k+1}\right)-c\left(t_{*}^{k}\right)}{\Delta t_{*}}= & \frac{3}{2} \mathbb{D}\left(t_{*}^{k}\right)-\frac{1}{2} \mathbb{D}\left(t_{*}^{k-1}\right) \\
& +\left(1+a_{k}\right) \mathbb{H}\left(t^{n}\right)-a_{k} \mathbb{H}\left(t^{n-1}\right) .
\end{aligned}
$$

Here, $\mathbb{D}=D \nabla^{2} c$ and $\mathbb{H}=(\mathbf{u} \cdot \nabla) c+W$ represent the diffusion term and the sum of the convection and reaction terms, respectively, and $a_{k}=(k+1 / 2) / K$ is an interpolation coefficient. To minimize numerical under/overshooting of $c$ value outside $[0,1]$ associated with center difference schemes, the convection term in Eq. (3) is discretized using a fifth order Weighted Essentially Non-Oscillatory (WENO) scheme, ${ }^{40}$ whereas all other spatial terms are discretized using sixth order center schemes.

The constant-density flow solver is largely identical to the solver used by us ${ }^{33,34}$ earlier, but the multi-grid solver ${ }^{41}$ for the constant-coefficient Poisson equation with periodic boundaries is replaced with an accurate spectrum solver using an open source, parallel version of FFTW3 (mpi-fftw). The DNS code is implemented in a vector form enabling $1 \mathrm{D}, 2 \mathrm{D}$, and 3D simulations.

The initial turbulence field is generated by synthesizing prescribed Fourier waves ${ }^{42}$ with an initial r.m.s. velocity $u_{0}^{\prime}$ and a turbulence length scale $\Lambda_{0}=\Lambda / 4$. Following Lamorgese et al.,$^{43}$ the forcing function $\mathbf{f}(\mathbf{x}, t)=\sum_{\kappa} \hat{\mathbf{f}}_{\kappa}(t) \exp (i \kappa \cdot \mathbf{x})$ is invoked in order to maintain statically stationary turbulence. Here,

$$
\hat{\mathbf{f}}_{\kappa}(t)=\frac{\langle\varepsilon\rangle 1_{\kappa-\kappa_{r}(t)}}{\hat{\mathbf{u}}_{\kappa}(t) \cdot \hat{\mathbf{u}}_{\kappa}^{*}(t)} \hat{\mathbf{u}}_{\kappa}(t)
$$

is the Fourier mode of $\mathbf{f}$ in the wavenumber $\kappa$-space,

$$
\varepsilon=2 v S_{i j} S_{i j}=\frac{v}{2}\left(\frac{\partial u_{i}}{\partial x_{j}}+\frac{\partial u_{j}}{\partial x_{i}}\right)\left(\frac{\partial u_{i}}{\partial x_{j}}+\frac{\partial u_{j}}{\partial x_{i}}\right)
$$

is the dissipation rate, the bracket $\langle\cdot\rangle$ designates averaging over entire domain, and the summation convention applies to repeated indexes $i$ and $j$. The caret operator designates the complex Fourier mode $\hat{q}_{\kappa}(t)=\langle q(\mathbf{x}, t) \exp (-i \kappa \cdot \mathbf{x})\rangle$ for any $q$, superscript star denotes the complex conjugate, $1_{\kappa-\kappa_{r}}=1$ when $\kappa=$ $\kappa_{r}$ or vanishes otherwise, $\kappa_{r}=2 \pi m_{i} \Lambda_{i}^{-1}$ is a randomly selected (at each time step) non-zero wavenumber within a userspecified lower wavenumber band, i.e., $\left|\kappa_{r}\right| \leq \kappa_{f}, \mathbf{m}=\left(m_{x}\right.$, $\left.m_{y}, m_{z}\right)$ is a random integer vector, and $\boldsymbol{\Lambda}=\left(\Lambda_{x}, \Lambda_{y}, \Lambda_{z}\right)$ is the domain length vector (note that $\Lambda_{y}=\Lambda_{z}=\Lambda$ ).

By adopting the same forcing technique with $\kappa_{f} / \kappa_{0}=3$, where $\kappa_{0}=2 \pi / \Lambda$, the present authors ${ }^{33,34}$ showed that (i) the r.m.s. velocity was maintained as the initial value, i.e., $u^{\prime}=u_{0}^{\prime}$, (ii) the normalized averaged dissipation rate $\left(\Lambda_{0} / u_{0}^{\prime 3}\right)\langle\varepsilon\rangle$ fluctuated slightly above $3 / 2$ after a short period $\left(t<\tau^{0}=\Lambda_{0} / u_{0}^{\prime}\right)$ of rapid transition from the initial artificially synthesized flow to developed turbulence, (iii) the forced turbulence achieved statistical homogeneity and isotropy over the entire domain (see also Fig. 1), and (iv) the energy spectrum showed a range

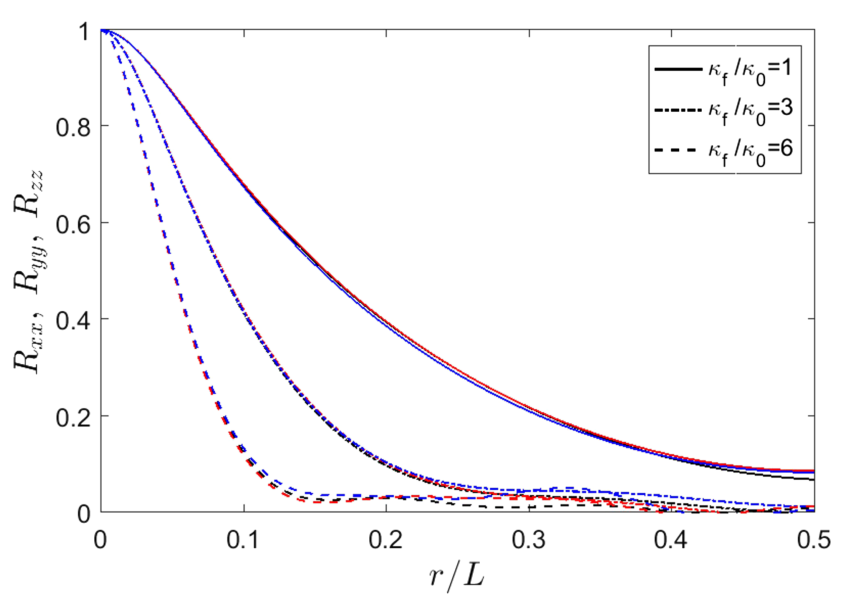

FIG. 1. Longitudinal auto-correlation functions $R_{11}^{u}$ (black), $R_{11}^{v}$ (red), and $R_{11}^{w}$ (blue) obtained from statistically stationary turbulence maintained using three forcing radii; $\kappa_{f} / \kappa_{0}=1$ (solid lines), 3 (dashed-dotted lines), and 6 (dashed lines). $R e_{0}=200$.

of the Kolmogorov scaling (-5/3) at $R e_{0}=u_{0}^{\prime} \Lambda_{0} / v=200$, see also Fig. 3.

In the present work, each of the generated turbulent fields is characterized with the longitudinal integral length scale $L_{11}=\int_{0}^{\Lambda / 2} R_{11}^{u}(r) d r \approx \int_{0}^{\Lambda / 2} R_{11}^{v}(r) d r \approx \int_{0}^{\Lambda / 2} R_{11}^{w}(r) d r$, the time scale $\tau_{t}=L_{11} / u^{\prime}$, the Reynolds number $R e_{t}=u^{\prime} L_{11} / v$, the dissipation rate $\overline{\langle\varepsilon\rangle}$ averaged both over the computational domain and time, the Kolmogorov length scale $\eta=\left(v^{3} / \overline{\langle\varepsilon\rangle}\right)^{1 / 4}$, and the Kolmogorov time scale $\tau_{\eta}=(v / \overline{\langle\varepsilon\rangle})^{1 / 2}$. These turbulence characteristics were calculated after the forced turbulence reached statistical stationarity, i.e., at $t>5 \tau_{t}^{0}$. The auto-correlation functions $R_{11}^{u}(r)$ $=\overline{\langle u(x, y, z) u(x+r, y, z)\rangle} / u^{\prime 2}, R_{11}^{v}(r)=\overline{\langle v(x, y, z) v(x, y+r, z)\rangle}$ $/ u^{\prime 2}$, and $R_{11}^{w}(r)=\overline{\langle w(x, y, z) v(x, y, z+r)\rangle} / u^{\prime 2}$ averaged over the transverse coordinates and time are plotted in Fig. 1, which further validate the isotropy of the generated turbulence.

Most present results were obtained at the same ratio of $\kappa_{f} / \kappa_{0}=3$. In such a case, three basic turbulence fields were generated by specifying three different values $R e_{0}=50,100$, or 200 of the "initial" turbulent Reynolds number, which was increased by increasing the domain size $\Lambda$. Three statistically same velocity fields were used in our previous DNS study ${ }^{33,34}$ of self-propagation of a passive, infinitely thin interface in a turbulent flow. Moreover, two more turbulence fields were generated by setting $\kappa_{f} / \kappa_{0}$ equal to 1 or $6\left(R e_{0}=200\right.$ in both cases) in order to change ratios of $L_{11} / \Lambda$ by retaining the same $u^{44}$ and $v$. A smaller forcing radius yields wider auto-correlation functions and, therefore, $\operatorname{larger} L_{11}$, see Fig. 1.

In the five aforementioned cases $\left(\kappa_{f} / \kappa_{0}=3\right.$ and $R e_{0}=50$, 100 , or $200 ; \kappa_{f} / \kappa_{0}=1$ and $R e_{0}=200 ; \kappa_{f} / \kappa_{0}=6$ and $R e_{0}=200$ ), the Kolmogorov length scale $\eta$ was on the order of the grid cell size $\Delta x$, see the second column in Table I in Sec. IV, thus, implying sufficient grid resolution. Capability of the used grids and numerical schemes for properly resolving the reaction wave was checked (i) in separate 1D simulations of laminar reaction waves, which were performed by running the same DNS code in the 1D case, and (ii) in recent 2D simulations of the development of the hydrodynamic instability 45,46 
of a laminar premixed flame in a wide computational domain. ${ }^{47}$ Nevertheless, in order to assess the sensitivity of computed results to numerical resolution, highly resolved simulations were also run by decreasing $\Delta x$ by a factor of four, see cases $\mathrm{H} 1$ and $\mathrm{H} 2$ in Table I.

In the present work, statistics of the $c(\mathbf{x}, t)$-fields were sampled at $t>3.5 \tau_{t}^{0}$, i.e., when the initial artificially synthesized velocity field reached a statistically stationary stage. The development of this velocity field to statistically stationary turbulence is illustrated in Fig. 2, which shows the temporary evolution of the simulated volume-averaged dissipation rate $\langle\varepsilon\rangle(t)$, while $u^{\prime}(t)$ is kept constant. At $t>4 \tau_{t}$ (note that the eddy-turn-over time $\tau_{t}=L_{11} / u^{\prime}$ is less than $\tau_{t}^{0}$ in all cases), the dissipation rates $\langle\varepsilon\rangle(t)$ are close to their fully developed values $\overline{\langle\varepsilon\rangle}$, which are averaged over time at $t>5 \tau_{t}^{0}$, with the amplitude of fluctuations in $\langle\varepsilon\rangle(t)$ with respect to $\overline{\langle\varepsilon\rangle}$ being low in all cases.

Figure 3 shows the statistically stationary spectra $E(|\kappa|)$ of the turbulent energy, computed using all above three forcing radii and at three $R e_{0}$. A wider range of $-5 / 3$ spectrum is obtained either for larger $R e_{0}$ or for smaller forcing radius $\left(\kappa_{f} / \kappa_{0}\right)$. It is worth stressing that the spectra simulated setting (i) $\kappa_{f} / \kappa_{0}=3$ and $R e_{0}=100$ and (ii) $\kappa_{f} / \kappa_{0}=6$ and $R e_{0}=200$ well match one another at large wavenumbers, cf. curves shown in green dotted-dashed and blue dashed lines. This matching is associated with equal values of $\kappa_{f} \Lambda_{0}$ $\propto\left(\kappa_{f} / \kappa_{0}\right) R e_{0}^{-1}$. Thus, the fields obtained at (i) $R e_{0}=100$ and $\kappa_{f} / \kappa_{0}=3$ and (ii) $R e_{0}=200$ and $\kappa_{f} / \kappa_{0}=6$ are characterized by almost equal $R e_{t}$ or $L_{11}$, but the ratio of $L_{11} / \Lambda$ is larger by a factor of about two in the former case. Accordingly, these two turbulence fields were used in order to gain insight into eventual influence of the relative width $\Lambda / L_{11}$ of the computational domain on obtained results, with all other things being approximately equal. As will be shown later, in the simulated constant-density reacting flows, such influence is weak because the auto-correlation functions vanish (if $\kappa_{f} / \kappa_{0} \geq 3$ ) at distance $r$ equal to halve the width of the computational domain, see Fig. 1.

In the present work, both fully developed and transient reaction waves were simulated starting from the pre-computed

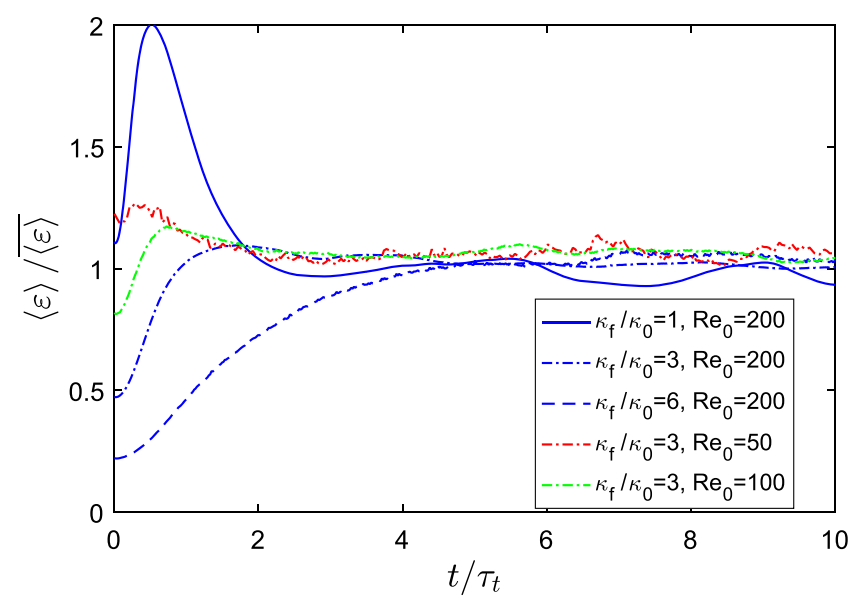

FIG. 2. Early $\left(0<t<10 \tau_{t}\right)$ evolution of the instantaneous, volume-averaged dissipation rate $(\langle\varepsilon\rangle)$ normalized using its fully developed value $\overline{\langle\varepsilon\rangle}$ averaged at $t>5 \tau_{t}^{0}$. Three different forcing radii and three different initial Reynolds numbers $R e_{0}$ are specified in legends.

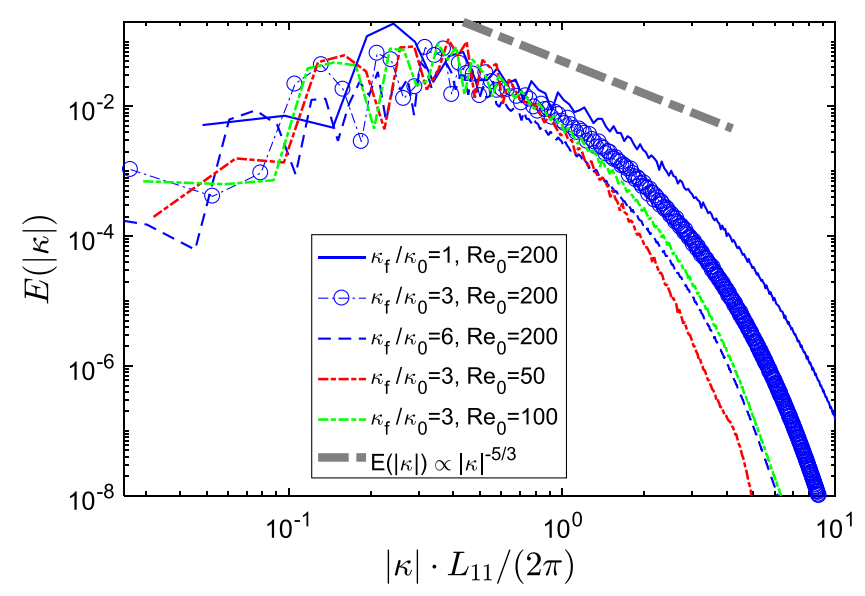

FIG. 3. Energy spectrums $E(\kappa)$ obtained from statistically stationary turbulence maintained using three forcing radii; $\kappa_{f} / \kappa_{0}=1$ (solid line), 3 (dotteddashed lines), and 6 (dashed line) at three different initial Reynolds numbers $R e_{0}=50$ (red), 100 (green), and 200 (blue).

laminar-wave profile of $c_{L}(\xi)$ with $d c_{L} / d \xi>0$. In order to study the fully developed turbulent reaction wave, a planar wave $c^{s}(x, 0)=c_{L}(\xi)$ was initially $(t=0)$ released at $x_{0}=\Lambda_{x} / 2$ such that $\int_{-\infty}^{0} c_{L}(\xi) d \xi=\int_{0}^{\infty}\left[1-c_{L}(\xi)\right] d \xi$ and $\xi=x-x_{0}$. Subsequently, evolution of this field $c^{s}(x, t)$ was simulated by solving Eq. (3). Computations of fully developed statistics with sampling every 100 time steps $\Delta t$ were started after the forced turbulence reached statistical stationarity at $t=t^{*}=6000 \Delta t>3.5 \tau_{t}^{0}$ and were performed over a time interval longer than 50 flow time scales $\tau_{t}^{0}=\Lambda_{0} / u^{\prime}$, with $\tau_{t}^{0}$ being on the order of, but larger than the eddy-turn-over time $\tau_{t}$ in all cases. For that purpose, the time-dependent mean value $\overline{q^{s}}(x, t)$ of a quantity $q^{s}(\mathbf{x}, t)$, e.g., $q^{s}=c^{s}$ and $q^{s}=W\left(c^{s}\right)$, was evaluated by averaging DNS data over transverse coordinates, followed by computing the fully developed profile of $\left\langle q^{s}\right\rangle(x)$ by averaging $\overline{q^{s}}(x, t)$ over time. Finally, $x$-coordinates were mapped to $\left\langle c^{s}\right\rangle(x)$-coordinates, as discussed elsewhere. ${ }^{34}$

In order to study transient turbulent reaction waves, the same pre-computed laminar-wave profiles $c_{L}(\xi)$ were simultaneously embedded into the turbulent flow in $M$ equidistantly separated planar zones centered around $x_{m} / \Lambda_{x}=(m-0.5) / M$, i.e., $c_{m}\left(x, t^{*}\right)=c_{L}\left(\xi_{m}\right)$, where coordinates $\xi_{m}=x-x_{m}$ were set using $\int_{-\infty}^{0} c_{L}\left(\xi_{m}\right) d \xi_{m}=\int_{0}^{\infty}\left[1-c_{L}\left(\xi_{m}\right)\right] d \xi_{m}$ and $m$ was an integer number $(1 \leq m<M=30)$. Subsequently, evolutions of $M$ transient fields $c_{m}^{t}(\mathbf{x}, t)$ were simulated by solving $M$ independent Eq. (3), with these fields affecting neither each other nor the turbulence in the studied case of $\rho=$ const. and $v=$ const. Accordingly, all $M$ transient fields $c_{m}^{t}(\mathbf{x}, t)$ were independent from each other, and the distance between iso-surfaces of two different transient fields did not affect computed results.

The transient simulations were run over $2 \tau_{t}^{0}$ before being reset. Subsequently, at $t=t^{*}+2 j \tau_{t}^{0}$, where $j \geq 1$, the flow was again populated by $M$ new profiles of $c_{L}\left(\xi_{m}\right)$ and the transient simulations were repeated. Time-dependent mean quantities $\overline{q^{t}}(x, t)$ were evaluated by averaging DNS data over transverse coordinates and over the entire ensemble $[m=1, \ldots, M$ and various time intervals $\left.t^{*}+2(j-1) \tau_{t}^{0} \leq t \leq t^{*}+2 j \tau_{t}^{0}\right]$ of the transient fields $q_{m}^{t}(x, t)$. Then, $x$-coordinates were mapped to $\overline{c^{t}}(x, t)$-coordinates, as discussed in detail elsewhere. ${ }^{34}$ 
Such a method (i.e., simulations of $M$ independent transient fields) significantly increased the sampling counts for calculating transient statistics and was already applied to studying self-propagation of an infinitely thin front in homogeneous isotropic turbulence. ${ }^{33,34}$ It is worth remembering, however, that such a method can only be used for simulating processes that do not affect the flow, e.g., a constant-density reaction wave addressed here. The transient simulations were performed only in the 15 basic cases, B1-B15, specified in Sec. IV because numerical integration of 31 independent Eq. (3) was sufficiently expensive.

Both fully developed and transient bulk consumption velocities were calculated by integrating the mean reaction rate along the normal to the mean reaction wave brush, i.e.,

$$
U_{T}^{s}=\int_{0}^{\Lambda_{x}}\left\langle W^{s}\right\rangle(x) d x
$$

and

$$
U_{T}^{t}(t)=\int_{0}^{\Lambda_{x}} \overline{W^{t}}(x, t) d x
$$

respectively.

Evolution of the major bulk characteristics (consumption velocity and mean wave brush thickness) of the transient fields $c_{m}^{t}(\mathbf{x}, t)$ was very similar in the present and earlier ${ }^{34}$ DNS studies. In particular, Figs. 14(a) and 15(a) in the cited paper ${ }^{34}$ indicate that the used time interval of $2 \tau_{t}^{0}$ is sufficient to investigate the largest part of the wave-development stage. For instance, at the end of this time interval, turbulent flame speed reached at least $80 \%$ of its fully developed value in the previous simulations. ${ }^{34}$ Similarly, in the present work, turbulent consumption velocity evaluated at the end of this time interval reached at least $80 \%$ of its fully developed value, which was obtained by simulating the propagation of the long-living reaction wave $c^{s}(\mathbf{x}, t)$.

\section{STUDIED CASES}

Four groups of characteristics of 45 studied cases are shown in Table I. The first group consists of two parameters $\eta / \Delta x$ and $\Lambda_{x} / \Lambda$, which characterize numerical meshes. In cases characterized by a high $u^{\prime} / S_{L} \geq 20$, a longer computational domain $\left(\Lambda_{x} / \Lambda=8\right)$ was used in order for the length of the entire reaction wave brush to be significantly less than $\Lambda_{x}$ always. With exception of cases $\mathrm{H} 1$ and $\mathrm{H} 2$, which were designed to check numerical resolution, all numerical meshes shared the same spatial step $\Delta x$, but the ratio of $\eta / \Delta x$ was varied due to variations of turbulence length scales, as discussed below.

The second group consists of four parameters, relevant to setting the longitudinal integral length scale $L_{11}$ of turbulence ( $u^{\prime}$ was the same in all cases), which was varied in two ways. Typically, $L_{11}$ was increased by increasing the numbers $N$ and $N_{x}$ of cells in the transverse and axial directions, respectively, by a factor of two (or four). Accordingly, the width $\Lambda$ of the computational domain was increased by a factor of two (or four) when compared to the width $\Lambda_{256}$, associated with $N_{x}$ $=256$. However, the length scale $L_{11}$ was increased less ${ }^{33}$ due to a slight decrease in $L_{11} / \Lambda$ with $\Lambda$, cf. $L_{11} / \Lambda$ in cases
B1, B6, and B11. In six particular cases (L1-L5 and H5), the length scale $L_{11}$ was varied by changing the characteristic wavenumber $\kappa_{f} / \kappa_{0}$ of turbulence forcing, but retaining $N_{x}$ and $\Lambda$ unchanged when compared to cases B11-B15. The seventh column reports the ratio of the eddy-turn-over time to the Kolmogorov time scale.

The third group consists of three parameters relevant to setting key mixture characteristics such as $S_{L}$ and $\delta_{F}=D / S_{L}$. As noted in Sec. II, these laminar wave characteristics are controlled by the Zeldovich number $Z e$, diffusivity $D$, and reaction time scale $\tau_{R}$, with the wave time scale $\tau_{F}=\delta_{F} / S_{L}$ being directly proportional to $\tau_{R}$ if $Z e=$ const. Accordingly, the eighth and ninth columns in Table I report $Z e$ and the Schmidt number $S c=v / D$, respectively. Variations in $S c$ are required in order to vary $S_{L}$ by retaining the same $\delta_{F}$ and vice versa. The tenth column reports the Damköhler number $D a=\tau_{t} / \tau_{F}$ because (the lack of) variations in $D a$ indicate (the lack of) variations in $\tau_{R}$ provided that turbulence characteristics $\left(L_{11}\right.$ and $\tau_{t}$ ) are kept constant.

The fourth group consists of turbulent wave characteristics such as Karlovitz $K a=\tau_{F} / \tau_{\eta}$, Reynolds $R e_{t}=\left(u^{\prime} L_{11}\right) / v$, and Péclet $P e=u^{\prime} L_{11} / D$ numbers, the normalized r.m.s. velocity $u^{\prime} / S_{L}$ and length scale $L_{11} / \delta_{F}$, as well as normalized fully developed turbulent consumption velocities $U_{T}^{s} / u^{\prime}$ and $U_{T}^{s} / S_{L}$. Although the Péclet number is rarely used in papers on turbulent combustion, $P e$ is specified in Table I for the following reasons. It is hypothesized in the seminal work of Damköhler ${ }^{48}$ that, in small-scale $\left(L_{11} \ll \delta_{F}\right)$ turbulence, burning velocity can be evaluated by (i) invoking a theoretical expression for the laminar flame speed $S_{L}$ and (ii) substituting the molecular diffusivity $D$ with turbulent diffusivity $D_{T} \sim u^{\prime} L_{11}$. Moreover, Damköhler ${ }^{48}$ assumed that $D=v$ or $S c=1$ and arrived at $S_{T} \propto S_{L} R e_{t}^{1 / 2}$. Damköhler's expression, i.e., $S_{T} \propto S_{L} R e_{t}^{1 / 2}$, is widely used in the turbulent combustion literature because $S c$ retains approximately the same value close to 0.7 in various premixed turbulent flames. However, in the present study, $S c$ is varied, e.g., in order to vary $S_{L}$ by retaining the same $\delta_{F}$. Accordingly, the aforementioned Damköhler's hypothesis leads to $S_{T} \propto S_{L} P e^{1 / 2}$, thus, implying that the Péclet number may play a more important role than the Reynolds number if the Schmidt number is varied.

Furthermore, while $R e_{t} \propto K a^{2} D a^{2}$, the values of $R e_{t}$ are also reported to stress the following important point. In the premixed turbulent combustion literature, the term "intense turbulence" is often associated with a low $D a<1$ and a high $K a>1$ or $u^{\prime} / S_{L} \gg 1$. Therefore, many cases addressed in Table I are associated with highly turbulent combustion. On the contrary, in the fluid mechanics literature, the same term "intense turbulence" is associated with $R e_{t} \gg 1$. From this viewpoint, all cases addressed in Table I are associated with weak turbulence or even transition to turbulence. This limitation of the present (and the vast majority of other) combustion DNS should be borne in mind.

As shown in Table I, several sets of simulations were performed. First, cases $\mathrm{B} n$, where $1 \leq n \leq 15$ is an integer number, are characterized by the same $\delta_{F}$ and compose three sets, B1-B5, B6-B10, and B11-B15, characterized by three different $L_{11}$. In each set, all cases are characterized by the same $L_{11}$ (and, hence, the same $L_{11} / \delta_{F}$ ) but five different 
$u^{\prime} / S_{L}$. Accordingly, each set is designed to study the influence of $u^{\prime} / S_{L}$ on turbulent consumption velocity. In all five cases B1-B5, B6-B10, or B11-B15 that had different $S_{L}$ but the same $R e_{t}$ and the same $\kappa_{f} / \kappa_{0}$, the flow statistics were the same because a reaction wave did not affect turbulence in the case of constant $\rho$ and $v$. Moreover, cases $\mathrm{B} l, \mathrm{~B}(l+5)$, and $\mathrm{B}(l+10)$, where $1 \leq l \leq 5$ is an integer number, belong to three different sets and are characterized by the same $u^{\prime} / S_{L}$ but different $L_{11}$ (and, hence, different $L_{11} / \delta_{F}$ ). Therefore such three cases (jointly with one of the cases $\mathrm{L} l$, discussed later) offer an opportunity to study the influence of $L_{11} / \delta_{F}$ on $U_{t}$ at five different $u^{\prime} / S_{L}$.

Second, five cases L1-L5 are basically similar to cases B11-B15, but forcing was performed at different wavenumbers in cases $\mathrm{L} l$ and $\mathrm{B} n$. Therefore, cases $\mathrm{L} l$ and $\mathrm{B}(l+10)$ share the same computational domain, the same $u^{\prime} / S_{L}$, and the same $\delta_{F}$ but are characterized by different $L_{11} / \Lambda$ and, hence, by different $L_{11} / \delta_{F}$.

Third, while $\delta_{F}$ was the same in 20 cases B1-B15 and L1-L5, four sets D1-D4, D5-D7, D8-D9, and D10 were designed to vary both $\delta_{F}$ and $S_{L}$ by retaining the same (in each set) $S c$ and, hence, the same diffusivity $D$. Each D-set is complementary to a single reference basic case, i.e., the complementary B-case and D-cases share the same $L_{11}$, the same $R e_{t}$, the same $S c$, and the same $P e$, whereas $u^{\prime} / S_{L}$ are different. In particular, (i) the set D1-D4 is complementary to case B15, (ii) D5-D7 to B7, (iii) D8-D9 to B10, and (iv) D10 to B5. Such complementary cases offer the opportunity to straightforwardly study the dependence of a ratio of $U_{T}^{s} / S_{L}$ on the diffusivity $D$.

Fourth, four sets of T-cases were designed to vary $L_{11}, S_{L}$, and $\delta_{F}$ by retaining the same Damköhler number $D a$. Each Tset has a reference $\mathrm{B}$ or $\mathrm{D}$-case, characterized by the same $\mathrm{Da}$, e.g., (i) B1 and T1, (ii) B5 and T2, (iii) D2 and T3-T4, and (iv) B15 and T5-T6. Such complementary cases enable us to study the influence of $D a$ on a ratio of $U_{T}^{s} / u^{\prime}$. It is also worth noting that, in addition to these specially designed cases, there are four more pairs of cases that are occasionally characterized by approximately equal $D a$ for each pair. These are cases B4 and B10, B8 and K1, B14 and L5, and D1 and D6.

Fifth, as the aforementioned cases characterized by approximately the same $D a$ are also characterized by sufficiently close Karlovitz numbers $K a$, three sets of $\mathrm{K}$-cases were designed in order to retain approximately the same $D a$ but to substantially change $K a$ when compared to a reference $\mathrm{B}$, $\mathrm{D}$, or $\mathrm{T}$ case. These sets are (i) $\mathrm{B} 2$ and $\mathrm{K} 1-\mathrm{K} 2$, (ii) $\mathrm{T} 4$ and $\mathrm{K} 3$, and (iii) D9 and K4. It is worth noting that, in cases K3 and $\mathrm{K} 4$, the viscosity was increased by a factor of four when compared to a reference value set in all other cases. Accordingly, $R e_{t}$ is low in cases $\mathrm{K} 3$ and $\mathrm{K} 4$. Such complementary cases make it possible to compare effects of $\mathrm{Da}$ and $\mathrm{Ka}$ on a ratio of $U_{T}^{s} / u^{\prime}$. Moreover, these cases offer the opportunity to study the influence of $R e_{t}$ on $U_{T}^{s} / u^{\prime}$ because variations in $R e_{t} \propto K a^{2} D a^{2}$ are required in order to vary $K a$ by retaining the same $D a$.

Sixth, five H-cases were designed in order (i) to check the sensitivity of results to numerical resolution, cf. cases B5 and $\mathrm{H} 1$, or to a ratio of the length scale $L_{11}$ to the width $\Lambda$ of the computational domain, cf. cases B10 and H5, and (ii) to study the influence of Zeldovich numbers on the wave characteristics, cases $\mathrm{H} 2-\mathrm{H} 4$.

Comparison of results computed in cases B5 and $\mathrm{H} 1$ validates the simulations by showing that a decrease in $\Delta x$ by a factor of four (H1) very weakly affects $U_{T}^{s} / u^{\prime}=0.40$ and 0.41 , respectively. The difference is as small as $2.5 \%$.

Comparison of results computed in cases B10 and H5 validates the simulations by showing that a decrease in a ratio of $L_{11} / \Lambda$ by a factor of two (H5) weakly affects $U_{T}^{s}$.

Comparison of results computed in cases $\mathrm{H} 1$ and $\mathrm{H} 2, \mathrm{~T} 2$ and $\mathrm{H} 3$, or $\mathrm{D} 4$ and $\mathrm{H} 4$ shows that an increase in $\mathrm{Ze}$ by a factor of about three (H-cases) weakly affects $U_{T}^{s}$.

\section{RESULTS AND DISCUSSION}

Figure 4 shows the evolution of five transient $c_{m}^{T}(\mathbf{x}, t)$ fields in cases B15 $\left(u^{\prime} / S_{L}=10\right.$, left column $)$ and B12 $\left(u^{\prime} / S_{L}=1\right.$, right column), with $R e_{t}$ being equal to 86 in both cases. Comparison of the left and right columns in Fig. 4 shows that a lower laminar wave speed $S_{L}$ is associated with a more wrinkled surface of the reaction zone and a thicker mean turbulent wave brush that propagates at a lower speed. For instance, at $\left(t-t_{*}\right) / \tau_{t}=5$, see the bottom row, the reaction zone for the $c_{7}^{T}(\mathbf{x}, t)$-field is close to the left boundary of the computational domain in the case of $u^{\prime} / S_{L}=1$, see image (j), but is sufficiently far from this boundary in the case of $u^{\prime} / S_{L}=10$, see image (i). While the five instantaneous $c$-iso-surfaces plotted in an image in Fig. 4 are different from each other, they show qualitatively similar appearances, thus, supporting the use of multiple $c_{m}^{T}(\mathbf{x}, t)$-fields to achieve more sampling for computing converged transient statistics.

\section{A. Fully developed turbulent consumption velocity}

Computed fully developed turbulent consumption velocities normalized using $u^{\prime}$ and $S_{L}$ are reported in the two right columns in Table I. Experimental data on turbulent burning velocities and flame speeds are commonly fitted using powerlaw dependencies of $U_{T}$ or $S_{T}$ on $u^{\prime}, S_{L}$, and $L_{11} / \delta_{F}$, with various models also yielding dependencies of such a kind. Accordingly, the same type of fitting was applied to the present DNS data on $U_{T}^{s}$. More specifically, the data were approximated with $Y=B X^{b}$ using least square fits, with $Y$ being substituted with $U_{T}^{s} / u^{\prime},\left(U_{T}^{s}-S_{L}\right) / u^{\prime}, U_{T}^{s} / S_{L}$, or $\left(U_{T}^{s}-S_{L}\right) / S_{L}$ and $X$ being substituted with $K a, R e_{t}$, or $\left(u^{\prime} / S_{L}\right) \cdot\left(L_{11} / \delta_{F}\right)^{d}$. For $X=\left(u^{\prime} / S_{L}\right) \cdot\left(L_{11} / \delta_{F}\right)^{d}$, the power exponent $d$ was varied from -32 to 32 with a step of 0.01 , the coefficients $B(d)$ and $b(d)$ were determined using least square fits for each value of $d$, and the final values of $d^{*}, B\left(d^{*}\right)$, and $b\left(d^{*}\right)$ were selected by maximizing a coefficient of determination, ${ }^{49}$ evaluated as follows:

$$
R^{2}=1-\left[\sum_{j=1}^{N}\left(Y_{j}-B X_{j}^{b}\right)^{2}\right]\left[\sum_{j=1}^{N}\left(Y_{j}-\frac{1}{N} \sum_{k=1}^{N} Y_{k}\right)^{2}\right]^{-1} .
$$

Here, $N=45$ is the number of simulated cases. It is worth noting that the subtraction of $S_{L}$ from $U_{T}^{S}$ substantially increased scatter of the DNS data around fitting curves. A similar trend was also observed ${ }^{4}$ when processing various experimental databases on $U_{T}$ and $S_{T}$. 

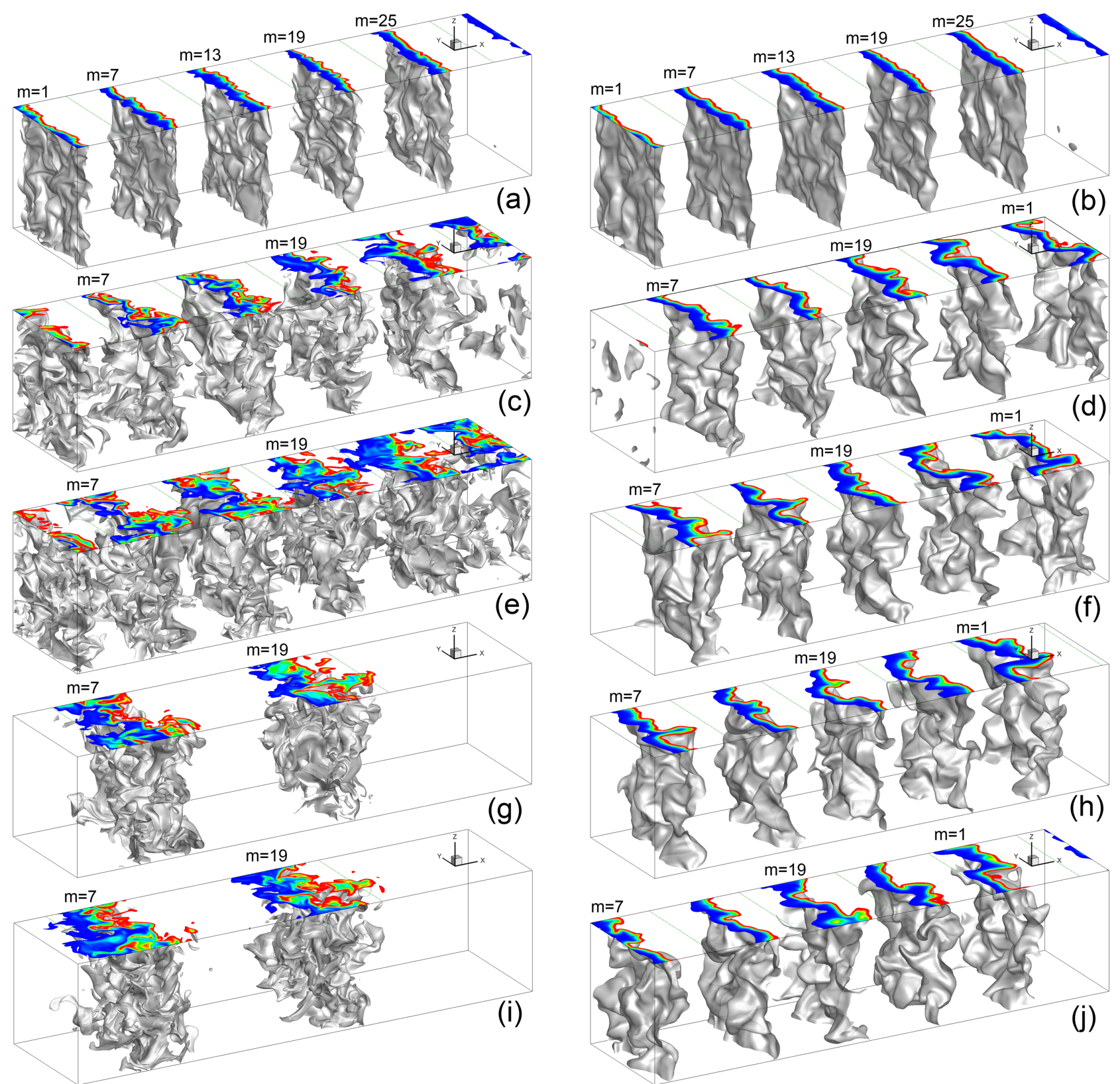

FIG. 4. Five (or sometimes two for clear visualization) independent $c_{m}^{t}(\mathbf{x}, t)$-fields obtained in the DNS at $\left(t-t_{*}\right) / \tau_{t}=1.0$ [(a) and (b)], 2.0 [(c) and (d)], 3.0 [(e) and (f)], $4.0\left[(\mathrm{~g})\right.$ and (h)], and $5.0\left[(\mathrm{i})\right.$ and (j)], $R e_{t}=86, \tau_{t}=0.4 \tau_{t}^{0}$, and $u^{\prime} / S_{L}=10$ [i.e., case B15, left column, i.e., (a), (c), (e), (g), and (i)] or 1.0 [case B12, right column, i.e., (b), (d), (f), (h), and (j)]. Grey iso-surfaces are associated with the peak reaction rate. Top-sliced planes are colored from blue to red in order to represent an increase in the local $c_{m}^{t}$ from zero to unity. All $c_{m}^{t}(\mathbf{x}, t)$-fields start as perfectly flat planes and propagate to the left until rewinding to the right due to periodicity.

Such an analysis of the DNS data has shown that the coefficient $R^{2}$ is most close to unity in the following two cases: (i) $Y=U_{T}^{s} / S_{L}, X=\left(u^{\prime} / S_{L}\right) \cdot\left(L_{11} / \delta_{F}\right)^{0.98}, B=0.89, b=0.51$, and $R^{2}=0.996$ and (ii) $Y=U_{T}^{s} / u^{\prime}, X=K a, B=2.24, b=-0.495$, and $R^{2}=0.930$. These two fits are very close to the following scaling expressions:

$$
\begin{gathered}
U_{T}^{s} \propto u^{\prime} \sqrt{D a} \propto S_{L} \sqrt{P e}, \\
U_{T}^{s} \propto u^{\prime} K a^{-0.5} \propto u^{\prime} \sqrt{D a} R e_{t}^{-1 / 4},
\end{gathered}
$$

respectively. It is worth noting that, first, Eq. (11) can be written in two equivalent forms, i.e., $\left(U_{T}^{s} / S_{L}\right)^{2}$ is proportional to the Péclet number Pe or $\left(U_{T}^{s} / u^{\prime}\right)^{2}$ is proportional to the Damköhler number $D a$. Second, the major difference between Eqs. (11) and (12) consists of a factor of $R e_{t}^{-1 / 4}$, which was varied in a sufficiently narrow range (from 2.2 to 3.5 if case $\mathrm{K} 4$ is skipped) under conditions of the present DNS.

Figure 5 shows that the DNS data are very well approximated with Eq. (11). At first glance, these data fully support the scaling of $U_{T}^{s} \propto S_{L} \sqrt{P e} \propto S_{L} \sqrt{R e_{t} S c}$. In the case of constant $S c=O(1)$, the scaling was theoretically predicted both in the distributed combustion regime associated with a low $\mathrm{Da}^{48}$ and the flamelet combustion regime associated with a high $D a{ }^{50}$ However, the inspection of the DNS data casts certain doubts on the universal validity of the scaling. 

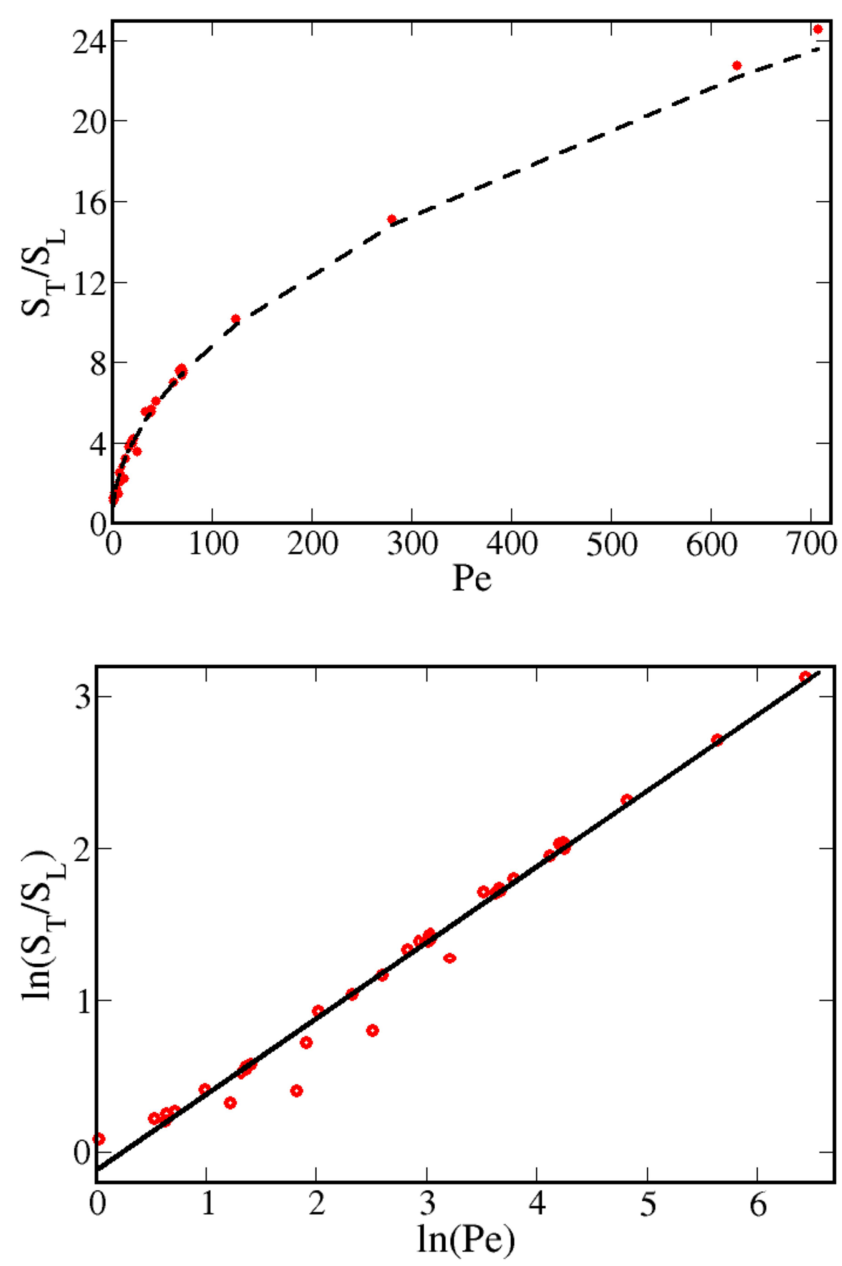

FIG. 5. Dependencies of $U_{T}^{s} / S_{L}$ on the Péclet number, reported in two different formats. DNS data are plotted in red symbols. Black lines show $U_{T}^{S} \propto S_{L} \sqrt{P e}$.

Indeed, as already noted earlier, our previous DNS study $^{33,34}$ of self-propagation of an infinitely thin interface in a turbulence field with the same statistical properties has yielded $U_{T}^{s} \propto u^{\prime}$. Therefore, comparison of the present and earlier DNS data implies that Eq. (11) should not be extrapolated to the limit case of infinitely large $L_{11} / \delta_{F} \rightarrow \infty$.

To assess such a hypothesis, we applied power-law fitting $U_{T}^{s} / u^{\prime} \propto\left(L_{11} / \delta_{F}\right)^{\beta}$ to DNS data obtained for a set of four reaction waves $\mathrm{B} l, \mathrm{~B}(l+5), \mathrm{B}(l+10)$, and $\mathrm{L} l$, characterized by the same $u^{\prime}$, the same $S_{L}$, but four different $L_{11} / \delta_{F}$. Results obtained for five such sets $(1 \leq l \leq 5)$ and plotted in Fig. 6(a) show that the magnitude $|\beta|$ of the power exponent is decreased when $u^{\prime} / S_{L}$ is decreased and, hence, $D a$ is increased. At the lowest value of $u^{\prime} / S_{L}$, see triangles, $|\beta|$ is as low as 0.18 . Thus, at low $u^{\prime} / S_{L}$ and large $D a$, the power exponent obtained in the present study becomes sufficiently close to the power exponent $\beta=0$ associated with the earlier study ${ }^{33,34}$ of self-propagation of an infinitely thin interface in a turbulence field with the same statistical properties.

Figure 6(a) indicates that if a dependence of $U_{T}^{s} / u^{\prime}$ on $L_{11} / \delta_{F}$ is approximated invoking a power law fitting within a bounded domain of $u^{\prime} / S_{L}$, the obtained power exponent $\beta$ is varied when the domain of $u^{\prime} / S_{L}$ is varied. Such a behavior of $\beta$ is fully consistent with the strong scatter of the fitted values of the discussed power exponent, reported in the literature. ${ }^{4}$ (a)

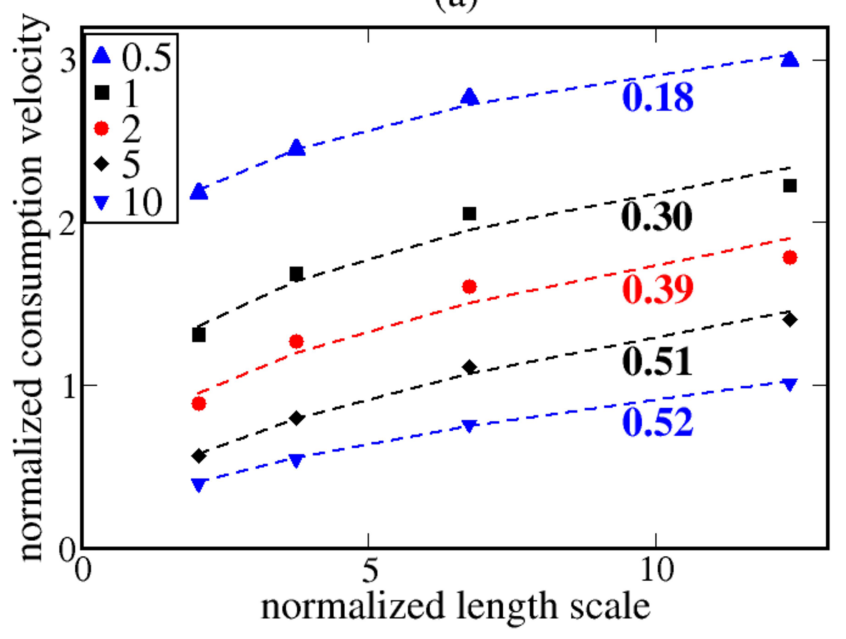

(b)

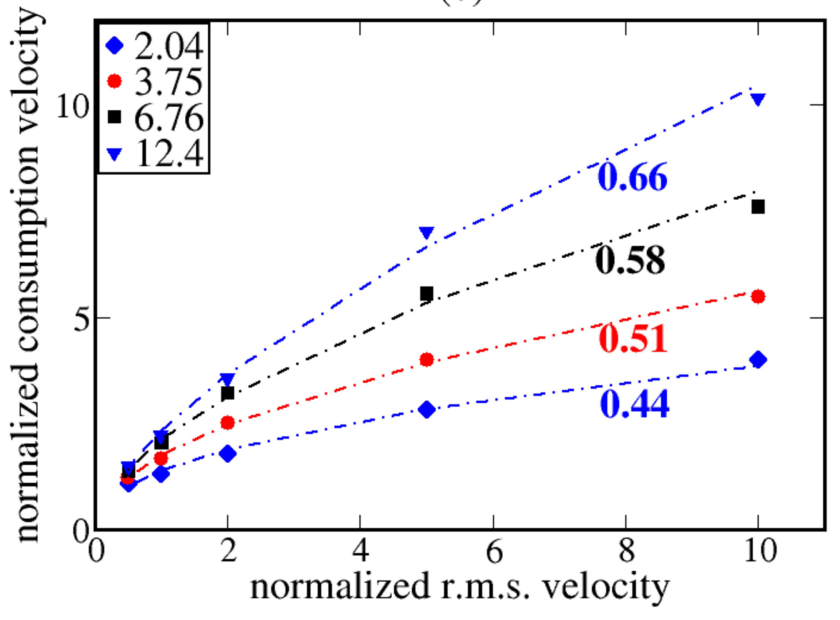

FIG. 6. (a) Approximation (lines) of DNS data (symbols) on $U_{T}^{s} / u^{\prime}$ using power-law fitting $U_{T}^{s} / u^{\prime} \propto\left(L_{11} / \delta_{F}\right)^{\beta}$, with the power exponent $\beta$ being specified near each curve. Each set of the data is characterized by the same $u^{\prime} / S_{L}$ specified in legends. (b) Approximation (lines) of DNS data (symbols) on $U_{T}^{s} / S_{L}$ using power-law fitting $U_{T}^{s} / S_{L} \propto\left(u^{\prime} / S_{L}\right)^{\alpha}$, with the power exponent $\alpha$ being specified near each curve. Each set of the data is characterized by the same $L_{11} / \delta_{F}$ specified in legends.

While an increase in $\beta$ with increasing $u^{\prime} / S_{L}$ has not yet been shown in the literature, to the best of the present authors' knowledge, this trend is not surprising and could be attributed to the change of the governing physical mechanism of the influence of turbulence on a reaction wave. For instance, the widely used regime diagrams of premixed turbulent combustion ${ }^{7,30-32}$ highlight different physical mechanisms in different ranges of $u^{\prime} / S_{L}$ and $L_{11} / \delta_{F}$. In particular, Damköhler ${ }^{48}$ considered two limiting cases of $\delta_{F} \ll \eta$ (and, hence, $K a \ll 1$ ) and $L_{11} \ll \delta_{F}$ (and, hence, $D a \ll 1$ if $u^{\prime} / S_{L} \geq 1$ ) and obtained the following scaling laws: $S_{T}=S_{L}+u^{\prime}$ and $S_{T}=S_{L} \sqrt{R e_{t}}$, respectively. Under conditions of $S c=1$, addressed by Damköhler, ${ }^{48}$ the latter scaling reads $S_{T} \propto S_{L} \sqrt{P e}$. Therefore, in the two limiting cases, $\beta=0$ and 0.5 , respectively. Thus, the theory by Damköhler ${ }^{48}$ predicts an increase in $\beta$ from zero to 0.5 with decreasing $D a$ or increasing $K a$. The present DNS data do show such an increase because an increase in $u^{\prime} / S_{L}$ is accompanied with an increase in $K a$ and a decrease in $D a$ in 20 cases addressed in Fig. 6(a). The discussed variations 
in $\beta$ with decreasing $D a$ and increasing $K a$ may substantially contribute to the strong scatter ${ }^{4}$ of the values of this power exponent, obtained by fitting various experimental databases on $S_{T}$ or $U_{T}$.

Results of a similar study of dependence of $U_{T}^{s} / S_{L}$ on $\left(u^{\prime} / S_{L}\right)^{\alpha}$ are reported in Fig. 6(b). We applied power-law fitting $U_{T}^{s} / S_{L} \propto\left(u^{\prime} / S_{L}\right)^{\alpha}$ to DNS data obtained for four sets (B1-B5, B6-B10, B11-B15, and L1-L5) of cases, with all reaction waves in each set being characterized by the same $L_{11}$, the same $\delta_{F}$, but five different $u^{\prime} / S_{L}$. Trends shown in Fig. 6(b) are qualitatively similar to trends shown in Fig. 6(a) (e.g., if $L_{11} / \delta_{F}$ and, hence, $D a$ is increased, the fitted $\alpha$ becomes more close to $\alpha=1$ obtained in the earlier studies ${ }^{33,34}$ ), but the trends are significantly less pronounced in Fig. 6(b) when compared to Fig. 6(a). For instance, the fitted values of $\alpha$ are sufficiently close to 0.5 under conditions of the present study. Probably for this reason, the values of this power exponent, fitted to various experimental databases, are substantially less scattered ${ }^{4}$ when compared to similarly fitted values of $\beta$ in $U_{T}^{s} / u^{\prime} \propto\left(L_{11} / \delta_{F}\right)^{\beta}$.

To gain better insight into eventual dependence of the power exponents on the Damköhler and Karlovitz numbers, the following investigation was performed. First, the array of 45 values of $U_{T}^{s}$, obtained in the DNS, was re-arranged starting from the case characterized by the highest $D a$ (case L1) and ending with the case characterized by the lowest $D a$ (case D9), i.e., $D a_{i} \leq D a_{i-1}$, where $1<i \leq 45$. Second, two truncated arrays of the data, i.e., $i_{1} \leq i \leq 45$ and $1 \leq i \leq i_{2}$, with $1 \leq i_{1} \leq 42$ and $4 \leq i_{2} \leq 45$, were fitted using either $U_{T}^{s} / u^{\prime}$ $=B D a^{b}$, see Eq. (11), or $U_{T}^{s} / u^{\prime}=B K a^{b}$, see Eq. (12). Third, the obtained dependencies of $R^{2}$ and $b$ on the boundary values $D a_{i_{1}}$ and $D a_{i_{2}}$ of the Damköhler number were analyzed. A similar study was also performed by re-arranging the same DNS data based on the Karlovitz number, i.e., $K a_{1}=0.36$ $<\cdots \leq K a_{i} \leq K a_{i+1} \leq \cdots<K a_{45}=587$. It is worth noting that both $D a_{1}=24.7$ and $K a_{1}=0.36$ correspond to the same case L1 and both $D a_{45}=0.01$ and $K a_{45}=587$ correspond to the same case D9.

Typical results of such studies are plotted in Figs. 7 and 8. First, Fig. 7(a) shows that the restriction of the fitted data to large values of $D a \geq D a_{i_{2}}$ results in significantly decreasing power exponents in $U_{T}^{s} / u^{\prime}=B D a^{b}$ when compared to the value of $b$ obtained by processing the entire data set, cf. red circles at $D a>1$ with blue dashed line, respectively. This difference implies the dependence of the fitted $b$ on the analyzed range of $D a$ if the Damköhler number is sufficiently high. If the same data are restricted to low $D a \leq D a_{i_{1}}$, see black squares, variations in the power exponent are much less pronounced, thus, indicating a weaker dependence of the fitted $b$ on the analyzed range of $D a$ if the Damköhler number is sufficiently low.

Similarly, Fig. 8(a) shows that the power exponent in $U_{T}^{s} / u^{\prime}=B K a^{b}$ varies less if the DNS data are restricted to high $K a \geq K a_{i_{1}}$ (black squares), whereas variations in $b$ are significant at low Karlovitz numbers if the DNS data are restricted to $K a \leq K a_{i_{2}}$ (red circles).

Second, Fig. 7(b) shows that both a decrease in $D a_{i_{1}}$ and an increase in $D a_{i_{2}}$ make $R^{2}$ very close to unity, thus, indicating that the DNS data computed either at low $\left(D a \leq D a_{i_{1}}\right)$ or at (a)

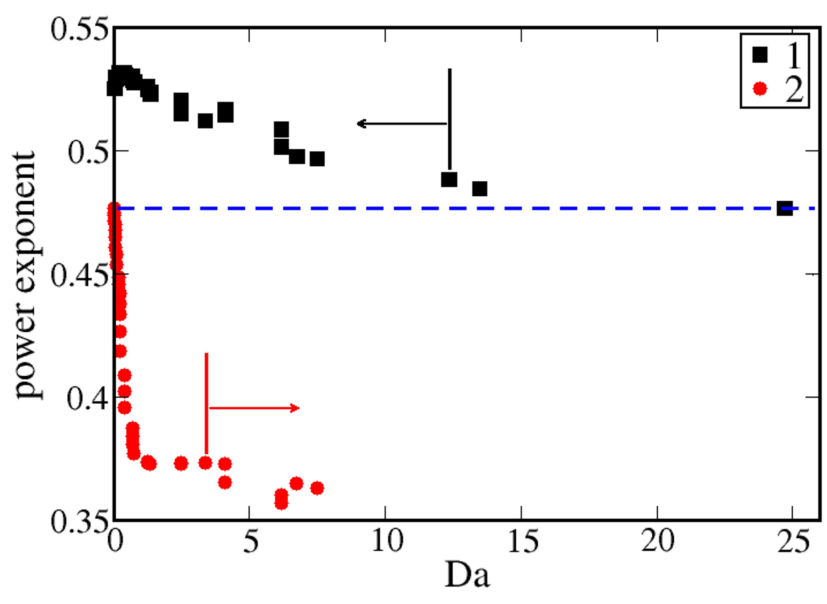

(b)

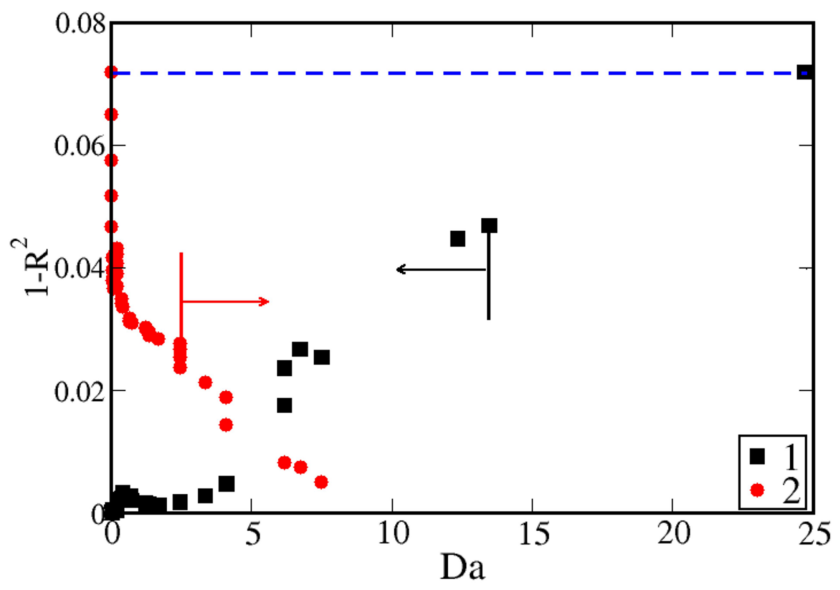

FIG. 7. (a) Power exponent in $U_{T}^{s} / u^{\prime}=B D a^{b}$ and (b) coefficient of determination vs. upper ( $D a_{i_{1}}$, black squares) and lower $\left(D a_{i_{2}}\right.$, red circles) boundaries of truncated arrays of the DNS data. Blue dashed lines show results obtained for all 45 DNS cases.

high ( $D a \geq D a_{i_{2}}$ ) Damköhler numbers are better fitted with $U_{T}^{s} / u^{\prime}=B D a^{b}$ when compared to the entire data set. This difference in the values of $R^{2}$ again implies that the data follow different scaling laws at low and at high $D a$.

Figure 8(b) shows that if the DNS data are fitted with $U_{T}^{s} / u^{\prime}=B K a^{b}$ and are restricted to high $K a \geq K a_{i_{1}}$ (black squares), then $R^{2}$ is very close to unity. If the same data are restricted to low $K a \leq K a_{i_{2}}$ (red circles), then $R^{2}$ is increased with decreasing $K a_{i_{2}}$, thus, indicating that $U_{T}^{s} / u^{\prime}=B K a^{b}$ fits the DNS data worse at low $K a$.

Finally, the dependence of the power exponents on $D a$ and $\mathrm{Ka}$ can also be indicated by straightforwardly applying Eqs. (11) and (12), respectively, to the entire data set. Indeed, results plotted in Fig. 9 show that both Eqs. (11) and (12) well approximate the data at low $\mathrm{Da}$ and $\mathrm{Ka}^{-1}$, respectively, but the scatter of the data is much more pronounced at $D a>4$ or $K a^{-1}>0.6$.

It is also worth noting that Fig. 6(a) shows that $|\beta|$ associated with $U_{T}^{s} / u^{\prime} \propto\left(\delta_{F} / L_{11}\right)^{\beta}$ is strongly decreased when $u^{\prime} / S_{L}$ is decreased from 5.0 to 0.5 , but the values of $\beta$ obtained at $u^{\prime} / S_{L}=5.0$ and 10.0 are almost equal to one another, cf. diamonds and triangles. Similarly, Fig. 6(b) indicates that $\alpha$ 
(a)

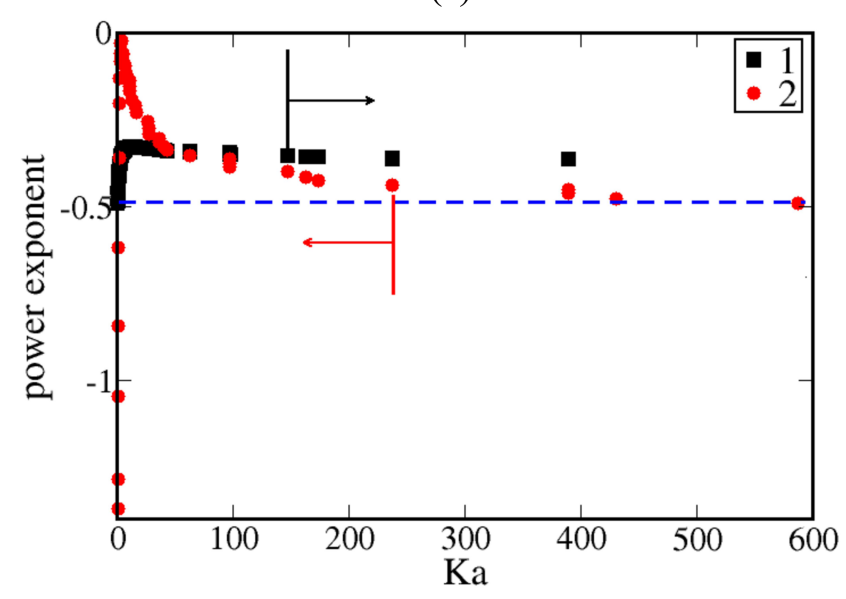

(b)

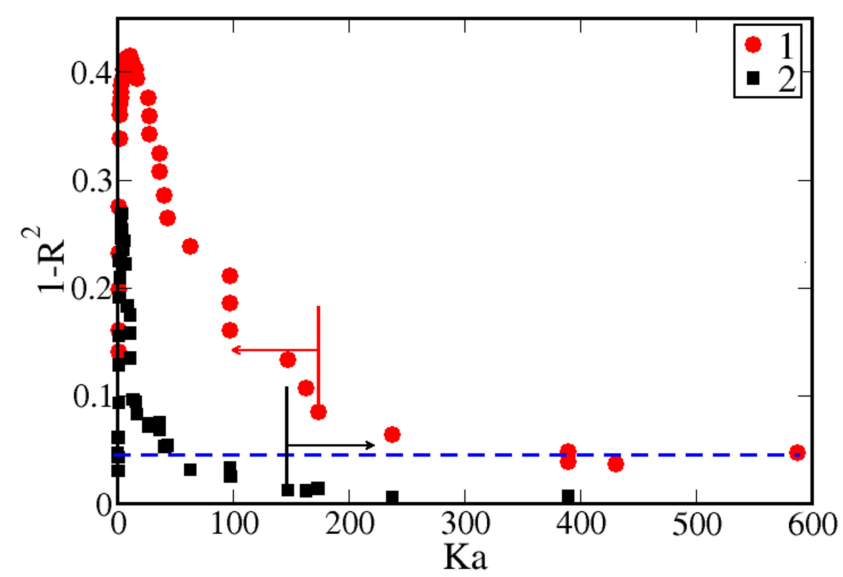

FIG. 8. (a) Power exponent in $U_{T}^{s} / u^{\prime}=B K a^{b}$ and (b) coefficient of determination vs. lower $\left(K a_{i_{1}}\right.$, black squares $)$ and upper $\left(K a_{i_{2}}\right.$, red circles $)$ boundaries of truncated arrays of the DNS data. Blue dashed lines show results obtained for all 45 DNS cases.

associated with $U_{T}^{s} / S_{L} \propto\left(u^{\prime} / S_{L}\right)^{\alpha}$ is approximately the same $(0.51 \pm 0.07)$ for three sets characterized by lower $L_{11} / \delta_{F}$ and, hence, $D a$. Based on these results, one may assume that dependencies of the two power exponents on $\mathrm{Da}$ (or $\mathrm{Ka}$ ) are leveled-off at $|\alpha|=|\beta|=0.5$, in line with Eq. (11), at sufficiently low (high) $\mathrm{Da}(\mathrm{Ka})$.

Such an assumption can be assessed by comparing values of $U_{T}^{s} / S_{L}$ computed at approximately equal $P e$. In six such sets of cases associated with low or moderate $D a$, i.e., (i) B1 and $\mathrm{T} 1$, (ii) B5, D10, and T2, (iii) B6, K1, and K2, (iv) B7 and D5D7, (v) B15 and D1-D4, and (vi) D8 and D9, approximately the same values of $U_{T}^{s} / S_{L}$ were computed for all cases within each set, in line with the discussed assumption.

If we accept Eq. (11) at sufficiently low $D a$, then a ratio of $U_{T}^{s} / u^{\prime}$ should solely be controlled by the Damköhler number. Indeed, Table I shows that approximately the same values of $U_{T}^{s} / u^{\prime}$ have been computed for all reaction waves within each of the 12 sets of cases characterized with approximately equal $\mathrm{Da}$, i.e., (i) $\mathrm{B} 1$ and $\mathrm{T} 1$, (ii) $\mathrm{B} 4$ and $\mathrm{B} 10$, (iii) $\mathrm{B} 8$ and $\mathrm{K} 1$, (iv) B14 and L5, (v) B15, T5, and T6, (vi) D1 and D6, (vii) D2, T3, and T4, (viii) D3, D7, and D8, (ix) D4 and D10, (xi) D9 and $\mathrm{K} 4$, and (xii) K1, K2, and B2. (a)

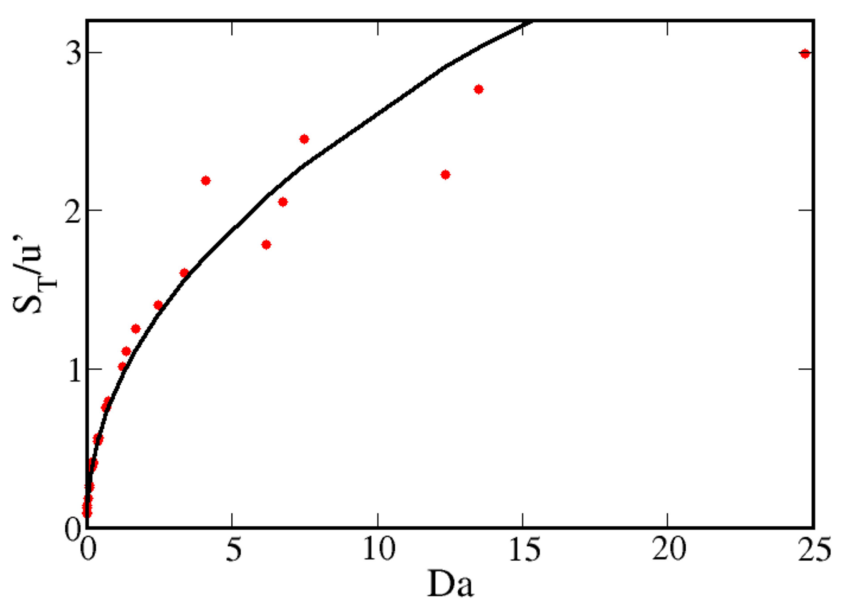

(b)

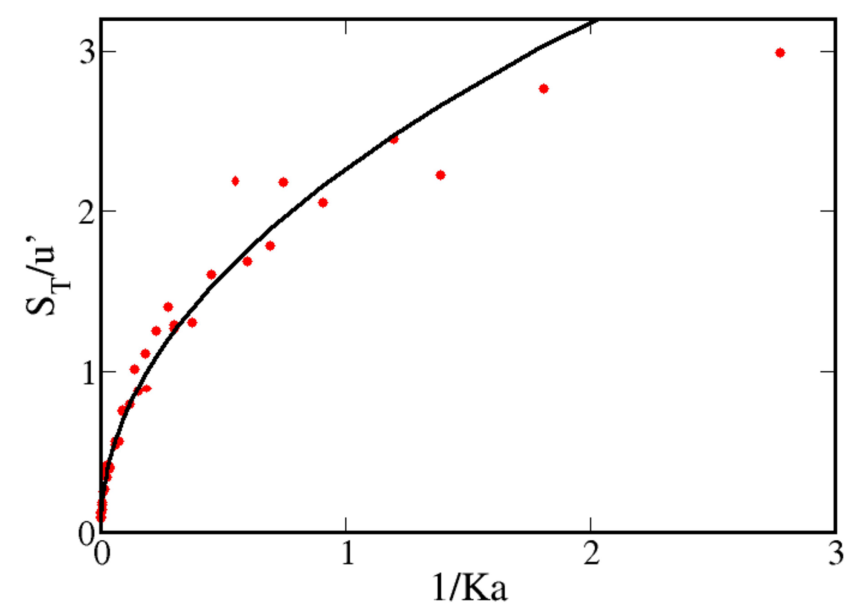

FIG. 9. Approximation of the DNS data with (a) $U_{T}^{s} \propto u^{\prime} \sqrt{D a}$ and (b) $U_{T}^{s} \propto$ $u^{\prime} / \sqrt{K a}$.

The above discussion implies that a ratio of $U_{T}^{s} / u^{\prime}$ is controlled by the Damköhler, rather than the Karlovitz number provided that $D a$ is sufficiently low. The same conclusion can be drawn by comparing values of $U_{T}^{s} / u^{\prime}$ computed in cases (i) B2, K1, and K2, (ii) T4 and K3, and (iii) D9 and K4. Within each of these three sets of cases, values of $D a$ are approximately the same, values of $K a$ are notably (up to $64 \%$, cf. cases B2 and K2) different, but, nevertheless, values of $U_{T}^{s} / u^{\prime}$ are approximately the same (difference is $9 \%$ or less).

The obtained better (worse) correlation of $U_{T}^{s} / u^{\prime}$ with $D a$ ( $K a$ ) implies that the smallest eddies of the Kolmogorov scale weakly affect $U_{T}$. In a recent DNS study of lean premixed hydrogen flames, Aspden ${ }^{51}$ found that "changes to viscosity made little difference to the general structure and appearance of the flame," and this finding also implies a minor influence of the Kolmogorov scales on $U_{T}$. In flames, such an effect could be attributed to the disappearance of the smallest turbulent eddies due to dilatation, ${ }^{52,53}$ but $\nabla \cdot \mathbf{u}=0$ in the constantdensity case studied by us. Accordingly, under conditions of the present simulations, the weak influence of the smallest eddies on $U_{T}$ stems from rapid smoothing of small-scale wrinkles of the reaction-zone surface due to molecular diffusion, as 
discussed in detail elsewhere ${ }^{54}$ Comparison of the efficiencies of the two physical mechanisms (dilatation and smoothing) appears to be an interesting task for future variable-density DNS of premixed turbulent combustion.

All in all, in line with the second $\left(L \ll \delta_{F}\right)$ hypothesis by Damköhler, ${ }^{48}$ the present DNS data support Eq. (11) but imply that it should not be used at sufficiently high $D a$. It is worth remembering that the validity of Eq. (11) at low $D a$ and high $K a$ was also shown in an earlier DNS study by Aspden et al. ${ }^{55,56}$

\section{B. Developing turbulent consumption velocity}

In order to gain insight into eventual variations of the scaling exponents $\alpha$ and $\beta$ during wave development, the present DNS data on the developing $U_{T}^{t}(t)$ were processed adopting the following two methods.

First, to mimic experiments with expanding statistically spherical flames, ${ }^{2,57-61}$ the mean non-dimensional reaction wave "radius" $\bar{r}_{f}$ normalized using the length scale $L_{11}$ was evaluated by numerically integrating the following equation:

$$
\tau_{t} \frac{d \bar{r}_{f}}{d t}=\frac{U_{T}^{t}(t)}{u^{\prime}},
$$

starting from $\bar{r}_{f}(0)=0.0$ and using the DNS data on $U_{T}^{t}(t)$. Subsequently, (i) a reference value $r^{*}$ was set, (ii) time required to reach this reference radius was found using $\bar{r}_{f}(t)=r^{*}$, and (iii) values of $U_{T}^{t}\left[\bar{r}_{f}(t)=r^{*}\right]$ obtained in the basic DNS cases B1-B15 at the same $\bar{r}_{f}(t)=r^{*}$, but different instants $t$, were fitted using Eq. (10). Such a method is associated with the aforementioned experiments because, in those experiments, flame images are recorded using the same window and, therefore, the flame speeds are evaluated in the same range of the flame radii independently of $u^{\prime}$, mixture composition, etc.

In the range of $0.25 \leq r^{*} \leq 1$, the best fit $\left(R^{2}=0.99\right)$ to the DNS data was obtained using $Y=U_{T}^{t} / u^{\prime}$ and $X=\left(u^{\prime} / S_{L}\right)$ - $\left(L_{11} / \delta_{F}\right)^{d}$. (Note that $d$ is defined in Sec. V A where it was also discussed that the same type of fitting yielded the lowest difference in $R^{2}$ and unity in the case of the fully developed $U_{T}^{s}$.) More specifically, in the range of $0.25 \leq r^{*} \leq 1$, the DNS data on $U_{T}^{t}\left[t\left(\bar{r}_{f}=r^{*}\right)\right]$ with the above best fit give $U_{T}^{t} \propto u^{\prime \alpha} S_{L}^{1-\alpha}\left(L_{11} / \delta_{F}\right)^{\beta}$, where $\alpha \approx 0.5$, but $\beta$ is increased with increasing $r^{*}$, see Table II. It is worth remembering that $\alpha \approx \beta \approx 0.5$ for the entire database on the fully developed $U_{T}^{s}$. Thus, in this particular case, the scaling exponent $\beta$ for $U_{T}^{t}$ as a function of $L_{11} / \delta_{F}$ changes substantially during the wave development, whereas the scaling exponent $\alpha$ for $U_{T}^{t}$ as a function of $u^{\prime} / S_{L}$ retains approximately the same value 0.5 .

It is also worth noting that the values of $\beta=0.22$ and 0.28 , obtained at $r^{*}=0.50$ and 0.75 , respectively, are sufficiently close to the power exponent 0.25 resulting for $L_{11} / \delta_{F}$ from the following scaling $U_{T} \propto u^{\prime} \mathrm{Da}^{1 / 4}$, which well fits ${ }^{2,4}$ to the most extensive experimental databases on $S_{T}$ and $U_{T}$ obtained from expanding statistically spherical turbulent premixed flames. However, values of $\alpha=0.5$ and 0.75 are substantially different in the present DNS and the cited experiments, respectively.

Second, values of $U_{T}^{t}(t)$ computed in the basic cases B1-B15 at the same non-dimensional time $\theta^{*}=t / \tau_{t}$ were fitted using Eq. (10) and varying $\theta^{*}$. Such a method is
TABLE II. Variations of scaling exponents during wave development.

\begin{tabular}{lllll}
\hline \multicolumn{5}{c}{ Fixed normalized mean wave radius $\bar{r}_{f}$} \\
\hline$r^{*}$ & 0.25 & 0.50 & 0.75 & 1.0 \\
$R^{2}$ & 0.99 & 0.99 & 0.99 & 0.99 \\
$\alpha$ & 0.5 & 0.5 & 0.5 & 0.5 \\
$\beta$ & 0.19 & 0.22 & 0.28 & 0.33 \\
\hline \multicolumn{5}{c}{ Fixed normalized time $\theta$} \\
\hline$\theta^{*}$ & 0.2 & 0.5 & 1.0 & \\
$R^{2}$ & 0.99 & 0.97 & 0.95 & \\
$\alpha$ & 0.05 & 0.20 & 0.35 \\
$\beta$ & 0 & 0 & 0 & \\
\hline \hline
\end{tabular}

associated with measurements performed in a statistically stationary Bunsen ${ }^{16,62-64}$ (rim-stabilized) or V-shaped ${ }^{65-67}$ (rod-stabilized) premixed turbulent flame at the same distance $x$ from the flame-stabilization zone. If mixture composition, $u^{\prime}$, and the bulk flow velocity $U$ are varied, but the ratio of $u^{\prime} / U$ retains the same value, then the normalized flamedevelopment time $(x / U) /\left(L_{11} / u^{\prime}\right)$ also retains the same value in such experiments.

At $\theta^{*}=0.2,0.5$, and 1.0, the maximal values of $R^{2}=0.99$, 0.97 , and 0.95 , respectively, were obtained for $Y=U_{T}^{t} / u^{\prime}$ and $X=u^{\prime} / S_{L}$. More specifically, at $0.2 \leq \theta^{*} \leq 1$, the DNS data on $U_{T}^{t}\left(\theta^{*}\right)$ are best fitted with $U_{T}^{t} \propto u^{\prime \alpha} S_{L}^{1-\alpha}\left(L_{11} / \delta_{F}\right)^{\beta}$, where $\beta$ vanishes and $\alpha$ is increased with increasing $\theta^{*}$, see Table II. A decrease in $\alpha$ or $\beta$ with decreasing $\theta^{*}$ is expected because $U_{T}$ should be sufficiently close to $S_{L}$ at low $\theta^{*}$.

The significant difference between the best fits to the DNS data on $U_{T}^{s}, U_{T}^{t}\left[t\left(\bar{r}_{f} \leq 1\right)\right]$, and $U_{T}^{t}\left(\theta^{*} \leq 1\right)$ implies that $S_{T}$ or $U_{T}$ is subject to significant transient effects during the premixed turbulent flame development. This result appears to be of substantial importance because a typical premixed turbulent flame investigated in a typical experiment is the developing flame, as discussed in detail elsewhere. ${ }^{4,10}$ Such transient effects should definitely be taken into account when comparing experimental data obtained using different measurement methods from different flames. Moreover, such transient effects should also be taken into account when comparing an analytical expression for the fully developed $U_{T}$ with experimental data obtained from developing flames.

\section{CONCLUSION}

The present DNS data show that, at sufficiently low Damköhler numbers $D a$, a ratio $U_{T}^{s} / u^{\prime}$ of fully developed turbulent consumption velocity to r.m.s. turbulent velocity is mainly controlled by $D a$ and scales as $\sqrt{D a}$, in line with the classical hypothesis by Damköhler. ${ }^{48}$ However, such a scaling should not be extrapolated to high $D a$.

The higher $D a$ (or $K a^{-1}$ ), the less pronounced dependence of $U_{T}^{s} / u^{\prime}$ on a ratio $L_{11} / \delta_{F}$ of integral turbulent length scale to the laminar wave thickness. This effect may contribute to significant scatter of scaling exponents $\beta$ obtained by fitting various experimental databases with $U_{T} \propto u^{\prime \alpha} S_{L}^{1-\alpha}\left(L_{11} / \delta_{F}\right)^{\beta}$.

Scaling laws for developing $U_{T}^{t}$ and fully developed $U_{T}^{s}$ can be substantially different. In particular, the aforementioned scaling exponents $\alpha$ and, especially, $\beta$ depend on the 
wave-development time and on a method used to evaluate the developing $U_{T}^{t}$. Such effects may also contribute to significant scatter of expressions for $U_{T}$ or $S_{T}$ as a function of $\left\{u^{\prime}\right.$, $\left.S_{L}, L_{11}, \delta_{F}\right\}$, obtained by parameterizing various experimental databases.

\section{ACKNOWLEDGMENTS}

R.Y. gratefully acknowledges the financial support from the Swedish Research Council (VR). A.N.L. gratefully acknowledges the financial support from the Chalmers Areas of Advance Transport and Energy and Combustion Engine Research Center (CERC). The authors are very grateful to Professor Vladimir Sabelnikov for fruitful discussion. The computations were performed using the computer facilities provided by the Centre for Scientific and Technical Computing at Lund University (Lunarc). Moreover, the computations were also performed on resources provided by the Swedish National Infrastructure for Computing (SNIC) at Beskow-PDC Center and Triolith-NSC Center.

${ }^{1}$ R. Abdel-Gayed, K. Al-Khishali, and D. Bradley, "Turbulent burning velocities and flame straining in explosions," Proc. R. Soc. A 391, 393-414 (1984).

${ }^{2}$ Ö. L. Gülder, "Turbulent premixed flame propagation models for different combustion regimes," Symp. (Int.) Combust. 23, 743-750 (1991).

${ }^{3}$ D. Bradley, "Problems of predicting turbulent burning rates," Combust. Theory Modell. 6, 361-382 (2002).

${ }^{4}$ A. N. Lipatnikov and J. Chomiak, "Turbulent flame speed and thickness: Phenomenology, evaluation, and application in multi-dimensional simulations," Prog. Energy Combust. Sci. 28, 1-74 (2002).

${ }^{5}$ V. L. Zimont and A. N. Lipatnikov, "A numerical model of premixed turbulent combustion," Chem. Phys. Rep. 14, 993-1025 (1995).

${ }^{6}$ H. G. Weller, G. Tabor, A. D. Gosman, and C. Fureby, "Application of a flame-wrinkling LES combustion model to a turbulent mixing layer," Symp. (Int.) Combust. 27, 899-907 (1998).

${ }^{7}$ N. Peters, Turbulent Combustion (Cambridge University Press, 2000).

${ }^{8}$ S. P. R. Muppala, N. K. Aluri, F. Dinkelacker, and A. Leipertz, "Development of an algebraic reaction rate closure for the numerical calculation of turbulent premixed methane, ethylene, and propane/air flames for pressures up to 1.0 MPa," Combust. Flame 140, 257-266 (2005).

${ }^{9}$ V. Molkov, F. Verbecke, and D. Makarov, "LES of hydrogen-air deflagrations in a 78.5-m tunnel," Combust. Sci. Technol. 180, 796-808 (2008).

${ }^{10}$ A. Lipatnikov, Fundamentals of Premixed Turbulent Combustion (CRC Press, Boca Raton, FL, 2012).

${ }^{11}$ I. G. Shepherd and L. W. Kostiuk, "The burning rate of premixed turbulent flames in divergent flows," Combust. Flame 96, 371-380 (1994).

${ }^{12}$ F. C. Gouldin, "Combustion intensity and burning rate integral of premixed flames," Symp. (Int.) Combust. 26, 381-388 (1996).

${ }^{13}$ A. N. Lipatnikov and J. Chomiak, "Application of the Markstein number concept to curved turbulent flames," Combust. Sci. Technol. 176, 331-358 (2004).

${ }^{14}$ B. R. Savarianandam and C. J. Lawn, "Burning velocity of premixed turbulent flames in the weakly wrinkled regime," Combust. Flame 146, 1-18 (2006).

${ }^{15}$ A. N. Lipatnikov and J. Chomiak, "Global stretch effects in premixed turbulent combustion," Proc. Combust. Inst. 31, 1361-1368 (2007).

${ }^{16}$ P. Venkateswaran, A. Marshall, D. H. Shin, D. Noble, J. Seitzman, and T. Lieuwen, "Measurements and analysis of turbulent consumption speeds of $\mathrm{H}_{2} / \mathrm{CO}$ mixtures," Combust. Flame 158, 1602-1614 (2011).

${ }^{17}$ S. Verma and A. N. Lipatnikov, "Does sensitivity of measured scaling exponents for turbulent burning velocity to flame configuration prove lack of generality of notion of turbulent burning velocity?" Combust. Flame 173, 77-88 (2016).

${ }^{18}$ J. F. Driscoll, "Turbulent premixed combustion: Flamelet structure and its effect on turbulent burning velocities," Prog. Energy Combust. Sci. 34, 91-134 (2008).
${ }^{19}$ B. Karlovitz, D. W. Denniston, and F. E. Wells, "Investigation of turbulent flames," J. Chem. Phys. 19, 541-547 (1951).

${ }^{20}$ A. C. Scurlock and J. H. Grover, "Propagation of turbulent flames," Symp. (Int.) Combust. 4, 645-658 (1953).

${ }^{21}$ H. Kolla, E. R. Hawkes, A. R. Kerstein, N. Swaminathan, and J. H. Chen, "On velocity and reactive scalar spectra in turbulent premixed flames," J. Fluid Mech. 754, 456-487 (2014).

${ }^{22}$ A. N. Lipatnikov, J. Chomiak, V. A. Sabelnikov, S. Nishiki, and T. Hasegawa, "Unburned mixture fingers in premixed turbulent flames," Proc. Combust. Inst. 35, 1401-1408 (2015).

${ }^{23}$ A. Y. Poludnenko, "Pulsating instability and self-acceleration of fast turbulent flames," Phys. Fluids 27, 014106 (2015).

${ }^{24}$ S. H. Kim, "Leading points and heat release effects in turbulent premixed flames," Proc. Combust. Inst. 36, 2017-2024 (2017).

${ }^{25}$ N. Fogla, F. Creta, and M. Matalon, "The turbulent flame speed for lowto-moderate turbulence intensities: Hydrodynamic theory vs. experiments," Combust. Flame 175, 155-169 (2017).

${ }^{26}$ K. N. C. Bray, “Turbulent transport in flames,” Proc. R. Soc. A 451, 231-256 (1995).

${ }^{27}$ V. R. Kuznetsov and V. A. Sabelnikov, Turbulence and Combustion (Hemisphere Publishing Corporation, 1985).

${ }^{28}$ A. N. Lipatnikov and J. Chomiak, "Effects of premixed flames on turbulence and turbulent scalar transport," Prog. Energy Combust. Sci. 36, 1-102 (2010).

${ }^{29}$ V. A. Sabelnikov and A. N. Lipatnikov, "Recent advances in understanding of thermal expansion effects in premixed turbulent flames," Annu. Rev. Fluid Mech. 49, 91-117 (2017).

${ }^{30}$ K. N. C. Bray, "Turbulent flows with premixed reactants," in Turbulent Reacting Flows (Springer-Verlag, Berlin, 1980), pp. 115-183.

${ }^{31}$ F. A. Williams, Combustion Theory (Benjamin/Cummings, Menlo Park, California, 1985).

${ }^{32}$ R. Borghi, "Turbulent combustion modeling," Prog. Energy Combust. Sci. 14, 245-292 (1988).

${ }^{33}$ R. Yu, A. Lipatnikov, and X.-S. Bai, "Three-dimensional direct numerical simulation study of conditioned moments associated with front propagation in turbulent flows," Phys. Fluids 26, 085104 (2014).

${ }^{34}$ R. Yu, X.-S. Bai, and A. N. Lipatnikov, "A direct numerical simulation study of interface propagation in homogeneous turbulence," J. Fluid Mech. 772, 127-164 (2015).

${ }^{35}$ R. Yu, J. Yu, and X.-S. Bai, "An improved high-order scheme for DNS of low Mach number turbulent reacting flows based on stiff chemistry solver," J. Comput. Phys. 231, 5504-5521 (2012).

${ }^{36}$ F. Zhang, R. Yu, and X.-S. Bai, "Detailed numerical simulation of syngas combustion under partially premixed combustion engine conditions," Int. J. Hydrogen Energy 37, 17285-17293 (2012).

${ }^{37}$ R. Yu and X.-S. Bai, "Direct numerical simulation of lean hydrogen/air autoignition in a constant volume enclosure," Combust. Flame 160, 1706-1716 (2013).

${ }^{38}$ J. Yu, R. Yu, X. Fan, M. Christensen, A. Konnov, and X.-S. Bai, "Onset of cellular flame instability in adiabatic $\mathrm{CH}_{4} / \mathrm{O}_{2} / \mathrm{CO}_{2}$ and $\mathrm{CH}_{4} /$ air laminar premixed flames stabilized on a flat-flame burner," Combust. Flame 160, 1276-1286 (2013).

${ }^{39}$ H. Carlsson, R. Yu, and X.-S. Bai, "Direct numerical simulation of lean premixed $\mathrm{CH}_{4} /$ air and $\mathrm{H}_{2}$ /air flames at high Karlovitz numbers," Int. J. Hydrogen Energy 39, 20216-20232 (2014).

${ }^{40}$ G.-S. Jiang and C.-W. Shu, "Efficient implementation of weighted ENO schemes,” J. Comput. Phys. 126, 202-228 (1996).

${ }^{41}$ R. Yu and X.-S. Bai, "A semi-implicit scheme for large eddy simulation of piston engine flow and combustion," Int. J. Numer. Methods Fluids 71, 13-40 (2013).

${ }^{42} \mathrm{R}$. Yu and X.-S. Bai, "A fully divergence-free method for generation of inhomogeneous and anisotropic turbulence with large spatial variation," J. Comput. Phys. 256, 234-253 (2014).

${ }^{43}$ A. G. Lamorgese, D. A. Caughey, and S. B. Pope, "Direct numerical simulation of homogeneous turbulence with hyperviscosity," Phys. Fluids 17, 015106 (2005).

${ }^{44} \mathrm{~V}$. Eswaran and S. B. Pope, "An examination of forcing in direct numerical simulations of turbulence," Comput. Fluids 16, 257-278 (1988).

${ }^{45}$ G. Darrieus, Propagation d' un front de flamme, unpublished work, presented at La Technique Moderne (Paris) in 1938 and at Congres de Mecanique Appliquee (Paris) in 1945 (1938).

${ }^{46}$ L. D. Landau, "On the theory of slow combustion," Acta Physicochim. USSR 19, 77-85 (1944). 
${ }^{47}$ R. Yu, X.-S. Bai, and V. Bychkov, "Fractal flame structure due to the hydrodynamic Darrieus-Landau instability,” Phy. Rev. E 92, 063028 (2015).

${ }^{48}$ G. Damköhler, "Der einfluss der turbulenz auf die flammengeschwindigkeit in gasgemischen," Ber. Bunsengesellschaft Phys. Chem. 46, 601-626 (1940).

${ }^{49}$ N. R. Draper and H. Smith, Applied Regression Analysis (WileyInterscience, 1998).

${ }^{50} \mathrm{~S}$. Chaudhuri, V. Akkerman, and C. K. Law, "Spectral formulation of turbulent flame speed with consideration of hydrodynamic instability," Phys. Rev. E 84, 026322 (2011).

${ }^{51}$ A. J. Aspden, "A numerical study of diffusive effects in turbulent lean premixed hydrogen flames," Symp. (Int.) Combust. 36, 1997-2004 (2017).

${ }^{52}$ T. Poinsot, D. Veynante, and S. Candel, "Quenching processes and premixed turbulent combustion diagrams," J. Fluid Mech. 228, 561-606 (1991).

${ }^{53}$ W. L. Roberts, J. F. Driscoll, M. C. Drake, and L. P. Goss, "Images of the quenching of a flame by a vortex-To quantify regimes of turbulent combustion," Combust. Flame 94, 58-69 (1993).

${ }^{54}$ R. Yu and A. N. Lipatnikov, "Direct numerical simulation study of statistically stationary propagation of reaction wave in homogeneous turbulence," Phys. Rev. E 96 (in press).

${ }^{55}$ A. J. Aspden, J. B. Bell, M. S. Day, S. E. Woosley, and M. Zingale, "Turbulence-flame interactions in type ia supernovae," Astrophys. J. 689, 1173-1185 (2008).

${ }^{56}$ A. J. Aspden, J. B. Bell, and S. E. Woosley, "Distributed flames in type ia supernovae," Astrophys. J. 710, 1654-1663 (2010).

${ }^{57}$ V. P. Karpov and E. S. Severin, "Effects of molecular-transport coefficients on the rate of turbulent combustion," Combust. Explosion Shock Waves 16, 41-46 (1980).

${ }^{58}$ D. Bradley, A. K. C. Lau, and M. Lawes, "Flame stretch rate as a determinant of turbulent burning velocity," Philos. Trans. R. Soc., A 338, 359-387 (1992).
${ }^{59}$ S. S. Shy, C. C. Liu, J. Y. Lin, L. L. Chen, A. N. Lipatnikov, and S. I. Yang, "Correlations of high-pressure lean methane and syngas turbulent burning velocities: Effects of turbulent Reynolds, Damköhler, and Karlovitz numbers," Proc. Combust. Inst. 35, 1509-1516 (2015).

${ }^{60}$ F. Wu, A. Saha, S. Chaudhuri, and C. K. Law, "Propagation speeds of expanding turbulent flames of $\mathrm{C}_{4}$ to $\mathrm{C}_{8} n$-alkanes at elevated pressures: Experimental determination, fuel similarity, and stretch-affected local extinction," Proc. Combust. Inst. 35, 1501-1508 (2015).

${ }^{61}$ J. Goulier, A. Comandini, F. Halter, and N. Chaumeix, "Experimental study on turbulent expanding flames of lean hydrogen/air mixtures," Proc. Combust. Inst. 36, 2823-2832 (2017).

${ }^{62}$ H. Kobayashi, T. Tamura, K. Maruta, T. Niioka, and F. A. Williams, "Burning velocity of turbulent premixed flames in a high-pressure environment," Symp. (Int.) Combust. 26, 389-396 (1996).

${ }^{63}$ P. Tamadonfar and O. L. Gülder, "Flame brush characteristics and burning velocities of premixed turbulent methane/air Bunsen flames," Combust. Flame 161, 3154-3165 (2014).

${ }^{64}$ T. M. Wabel, A. W. Skiba, J. E. Temme, and J. F. Driscoll, "Measurements to determine the regimes of premixed flames in extreme turbulence," Proc. Combust. Inst. 36, 1809-1816 (2017).

${ }^{65}$ S. Kheirkhah and O. L. Gülder, "Turbulent premixed combustion in Vshaped flames: Characteristics of flame front," Phys. Fluids 25, 055107 (2013).

${ }^{66}$ T. Sponfeldner, N. Soulopoulos, F. Beyrau, Y. Hardalupas, A. M. K. P. Taylor, and J. C. Vassilicos, "The structure of turbulent flames in fractaland regular-grid-generated turbulence," Combust. Flame 162, 3379-3393 (2015).

${ }^{67}$ A. A. Verbeek, P. A. Willems, G. G. M. Stoffels, B. J. Geurts, and T. H. van der Meer, "Enhancement of turbulent flame speed of V-shaped flames in fractal-grid-generated turbulence," Combust. Flame 167, 97-112 (2016). 